\title{
CONVERGENCE OF THE LAX-FRIEDRICHS SCHEME AND STABILITY FOR CONSERVATION LAWS WITH A DISCONTINUOUS SPACE-TIME DEPENDENT FLUX
}

\author{
KENNETH H. KARLSEN AND JOHN D. TOWERS
}

\begin{abstract}
We give the first convergence proof for the Lax-Friedrichs finite difference scheme for non-convex genuinely nonlinear scalar conservation laws of the form

$$
u_{t}+f(k(x, t), u)_{x}=0,
$$

where the coefficient $k(x, t)$ is allowed to be discontinuous along curves in the $(x, t)$ plane. In contrast to most of the existing literature on problems with discontinuous coefficients, our convergence proof is not based on the singular mapping approach, but rather on the div-curl lemma (but not the Young measure) and a Lax type entropy estimate that is robust with respect to the regularity of $k(x, t)$. Following [14], we propose a definition of entropy solution that extends the classical Kružkov definition to the situation where $k(x, t)$ is piecewise Lipschitz continuous in the $(x, t)$ plane. We prove stability (uniqueness) of such entropy solutions, provided that the flux function satisfies a so-called crossing condition, and that strong traces of the solution exist along the curves where $k(x, t)$ is discontinuous. We show that a convergent subsequence of approximations produced by the Lax-Friedrichs scheme converges to such an entropy solution, implying that the entire computed sequence converges.
\end{abstract}

\section{Contents}

1. Introduction

2. Assumptions and the Lax-Friedrichs scheme 4

3. A compensated compactness lemma $\quad 5$

4. Convergence analysis $r$

5. A Kružkov type entropy inequality 16

6. A uniqueness result 22

$\begin{array}{ll}\text { 7. A final remark about uniqueness } & 27\end{array}$

$\begin{array}{ll}\text { References } & 27\end{array}$

\section{INTRODUCTION}

The main part of this paper investigates the Lax-Friedrichs finite difference algorithm as it applies to the Cauchy problem for scalar conservation laws with the form

$$
u_{t}+f(k(x, t), u)_{x}=0, \quad u(x, 0)=u_{0}(x),
$$

where $(x, t) \in \mathbb{R} \times \mathbb{R}_{+} ; u(x, t)$ is the scalar unknown function; and $u_{0}, k, f(k, u)$ are given functions to be detailed later. Here it suffices to say that for the convergence analysis we need $k(x, t) \in$ $B V_{\text {loc }}\left(\mathbb{R} \times \mathbb{R}_{+}\right), u \mapsto f(k(x, t), u)$ genuinely nonlinear for a.e. $(x, t)$, and $u_{0}(x)$ bounded (see Section 2 for the complete list of assumptions).

The special feature of the problem studied herein is the nonlinear flux function $f(k(x, t), u)$ that depends explicitly on the spatial and temporal variables through a coefficient $k(x, t)$ that may be discontinuous. Problems like (1.1) occur in a variety of applications, and they have been widely studied in recent years, both from a mathematical and numerical point of view, at least

Date: June 3, 2004.

1991 Mathematics Subject Classification. 35L65, 35L45, 65M06, 65M12.

Key words and phrases. conservation law, discontinuous coefficient, nonconvex flux, Lax-Friedrichs difference scheme, convergence, compensated compactness, entropy condition, uniqueness.

Acknowledgment: This research was supported in part by the BeMatA program of the Research Council of Norway and the European network HYKE, funded by the EC as contract HPRN-CT-2002-00282. We thank Siddhartha Mishra for helping us to understand the entropy condition of [1]. 
when $k$ is independent of the time variable $t$, in which case (1.1) is equivalent to a $2 \times 2$ resonant (non-strictly hyperbolic) system of conservation laws:

$$
k_{t}=0, \quad u_{t}+f(k, u)_{x}=0 .
$$

Since there is generally no spatial total variation bound for $u$, the "singular mapping" approach has been used as the main analytical tool for proving convergence of various numerical schemes and thereby existence of weak solutions. The singular mapping approach to establishing compactness consists of transforming the numerical approximation $u^{\Delta}(x, t)$ via $z^{\Delta}(x, t)=\Psi\left(k(x, t), u^{\Delta}(x, t)\right)$, where $\Psi(k, u)$ is the so-called singular mapping. A singular mapping that is appropriate for the problem considered here is

$$
\Psi(k, u)=\int^{u}\left|f_{w}(k, w)\right| d w
$$

In many cases it is possible to show that the total variation of the transformed quantity $z^{\Delta}$ is bounded independently of the level of the discretization parameter $\Delta$. Helly's theorem then gives convergence (along a subsequence) of $z^{\Delta}$ as $\Delta \downarrow 0$. Since the continuous mapping $u \mapsto \Psi(k, u)$ is one-to-one, $u^{\Delta}$ also converges (along the same subsequence as $z^{\Delta}$ ).

Regarding previous work on problems like (1.1), we refer to [32, 20, 21, 9] for Glimm and Godunov schemes (based on (1.2) not (1.1)) and [8, 17, 18] for front tracking schemes (based on (1.2)). Appropriate scalar versions of the Godunov and Engquist-Osher schemes are analyzed in $[33,34,13]$, see also $[12,11]$ for some other scalar approximation schemes and an application of compensated compactness. We refer to introductory parts of $[12,13]$ for an overview of the activity on problems with discontinuous fluxes. A variety of results have been obtained recently also for a model of continuous sedimentation in ideal clarifier-thickener units. This model consists of a particular conservation law with two discontinuous coefficients. We refer to [2, 3] (and the references cited therein) for an overview of the mathematical and numerical results for this model.

Some time ago, Lin, Temple, and Wang [20] observed that a bound on the total variation (measured under the singular mapping), and thus convergence, had not yet been established for the $2 \times 2$ Lax-Friedrichs scheme, nor for any of the scalar schemes that apply to the version of (1.1) where the coefficient $k=k(x)$ is independent of time. Since then such bounds have been established for a number of scalar schemes, but the question of the Lax-Friedrichs scheme has been left unresolved. Numerical evidence indicates that the Lax-Friedrichs scheme is well-behaved on these problems (see, e.g., [2]). In fact Tveito and Winther [35] provide examples where the LaxFriedrichs scheme performs well while methods based on $2 \times 2$ Riemann solvers may fail. Based on this numerical evidence, we conclude that it is the singular mapping approach that is deficient at this point. This approach to convergence for scalar difference schemes depends strongly on the close functional relationship between the viscosity of the Engquist-Osher flux, the Kružkov entropy flux, and the singular mapping. This is true also for the Godunov scheme, where the proof depends on the fact that the Engquist-Osher flux is nearly identical to the Godunov flux when $f$ is concave. This lead us to believe that the singular mapping approach is not readily applicable to the Lax-Friedrichs scheme, and it motivated our interest in the compensated compactness approach $[23,24,25,30,31,7,4,22]$, which represents a departure from the singular mapping technique used in the literature cited above. Another aspect is that, possibly due to technical difficulties with the singular mapping approach, the case where the nonlinearity $u \mapsto f(k, u)$ is nonconvex has received less attention in the literature than the convex/concave case. An attractive feature of the compensated compactness approach is that no convexity condition is required for $u \mapsto f(k, u)$, but merely that $u \mapsto f(k(x, t), u)$ is "genuinely nonlinear" for a.e. $(x, t)$, i.e., for a.e. $(x, t)$ there are no intervals where $u \mapsto f(k(x, t), u)$ is linear. Also, sign changes of the discontinuous coefficient $k$ are handled without any special considerations. Sign changes are commonly ruled out $[17,18,16,33,34]$ due to added analytical technicalities with the singular mapping approach. Finally, being one of the points of this paper, the time dependent coefficient case can be treated without any new significant difficulties compared to the time independent case. Most of the previous literature assumes that the coefficient is time independent, but see the discussion below.

The present paper can be divided into three parts as follows: 
1. In Section 3 we prove a compensated compactness lemma in the spirit of Tartar [30, 31]. The proof is based on the div-curl lemma but does not rely on the Young measure. This is an advantage in the present general context since the fundamental theorem of Young measures applies most easily to functions $F(x, t, u)$ that are continuous in all the variables (herein we are interested in the $(x, t)$ discontinuous function $F(x, t, u)=f(k(x, t), u))$. Although we will omit the details, the compensated compactness lemma found herein can be formulated for problems with a vector-valued coefficient $k(x, t)=\left(k_{1}(x, t), \ldots, k_{M}(x, t), M \geq 1\right.$. Vector-valued coefficients occur naturally in certain applications, including models for continuous sedimentation, see $[2,3]$ and the references cited therein.

2. We use the compensated compactness lemma to prove convergence to a weak solution of the Lax-Friedrichs scheme as it applies to (1.1), thereby obtaining the first convergence result for the Lax-Friedrichs scheme as applied to conservation laws with a discontinuous flux. The details are presented in Sections 2 and 4. As a direct consequence we obtain an existence result for (1.1) with no assumptions on the convexity/concavity of $u \mapsto f(k, u)$ and/or sign changes in $k(x, t)$. In addition, more or less for free, our method of analysis allows for a time dependent coefficient. The time dependent case was treated only recently in [27]. The author proved existence of a unique and stable solution under the assumption that $u \mapsto f(k, u)$ is convex and $k(x, t)$ is piecewise smooth, i.e., $k(x, t)$ is allowed to be discontinuous along a finite number of curves in the $(x, t)$ plane. The method of proof differs from the singular mapping and compensated compactness approaches. It consists in proving convergence of the control theory representation of the unique viscosity solution of the corresponding Hamilton-Jacobi equation where the coefficient $k(\cdot, t)$ has been smoothed out via a standard mollifier. After the main results of this paper were obtained, we learned of a preprint [5] in which the authors use the singular mapping approach to prove convergence of a front tracking scheme for (1.1) when the coefficient $k(x, t)$ has a multiplicative space-time dependence $a(x) g(t)>0$, the nonlinearity $u \mapsto f(k, u)$ is concave, the mapping $k \mapsto f(k, u)$ is nondecreasing, and the initial function $u_{0}(x)$ is roughly speaking of bounded total variation. We would like to stress that the existence result given herein holds under conditions that are significantly more general than those needed for the existence results in $[27,5]$.

3. For a general class of problems with "piecewise smooth" coefficients and a possibly degenerate diffusion operator, the authors of [14] introduced a Kružkov type notion of an entropy solution and proved uniqueness of this solution. Uniqueness was proved for flux functions that satisfied a so called "crossing condition" and a technical condition regarding the existence of traces at the jump points in the coefficients. In [14] it was assumed that the discontinuous coefficients did not depend on the temporal variable $t$. The notion of entropy solution and the uniqueness result in [14] extend to time dependent coefficients. This extension is the topic of Sections 5 and 6 , see also Section 7 for a final remark about our entropy solution theory and its relation to [1, 10]. Regarding uniqueness of solutions to (1.1) when $k=k(x)$ is allowed to be discontinuous, some other results can be found in $[18,16,33,28]$. In Section 5 we prove that any limit of the Lax-Friedrichs scheme is an entropy solution. Consequently, whenever the uniqueness result applies, the whole sequence of Lax-Friedrichs approximate solutions converge to the entropy solution.

We close this introduction by making a few remarks on the usefulness of the Lax-Friedrichs scheme in applications. Roughly speaking, shock-capturing numerical schemes for hyperbolic problems can be classified as either central (Lax-Friedrichs type) schemes or upwind (Godunov type) schemes. A disadvantage with upwind schemes is that one needs the (exact or approximate) solution of the Riemann problem. In the 1990s the central schemes received much interest after the introduction of the second-order sequel to the Lax-Friedrichs scheme in [26]. The second order central scheme in [26] can be viewed as a direct extension of the first-order Lax-Friedrichs central scheme, in which the problem of excessive dissipation is resolved by reconstructing, in each time step, a MUSCL type piecewise-linear interpolant from the cell averages computed in the previous time step. The second order central scheme is as easy to use as the first order Lax-Friedrichs scheme (there is no need for a Riemann solver). We refer to the lecture notes [29] for a general overview of central schemes and their applications. In [2] we applied first and second order central schemes to (systems of) conservation laws with discontinuous coefficients modeling continuous sedimentation 
in ideal clarifier-thickener units, and we refer to that paper for numerical experiments illustrating the performance of the Lax-Friedrichs scheme studied herein.

\section{Assumptions and the LaX-Friedrichs scheme}

We begin by listing some assumptions on $u_{0}, k, f$ that are needed for the convergence analysis (more assumptions will be introduced in Section 5 for the definition of entropy solution).

Regarding the initial function we assume

$$
u_{0} \in L^{\infty}(\mathbb{R}), \quad a \leq u_{0}(x) \leq b \quad \text { for a.e. } x \in \mathbb{R} .
$$

For the discontinuous coefficient $k: \mathbb{R} \times \mathbb{R}_{+} \rightarrow \mathbb{R}$ we assume that

$$
k \in L^{\infty}\left(\mathbb{R} \times \mathbb{R}_{+}\right) \cap B V_{\text {loc }}\left(\mathbb{R} \times \mathbb{R}_{+}\right), \quad \alpha \leq k(x, t) \leq \beta \quad \text { for a.e. }(x, t) \in \mathbb{R} \times \mathbb{R}_{+} .
$$

We recall that $B V_{\text {loc }}\left(\mathbb{R} \times \mathbb{R}_{+}\right)$denotes the set of $L_{\text {loc }}^{1}\left(\mathbb{R} \times \mathbb{R}_{+}\right)$functions $z(x, t)$ for which the partial derivatives $z_{x}$ and $z_{t}$ are bounded Radon measures on compact subsets of $\mathbb{R} \times \mathbb{R}_{+}$.

Regarding the flux function $f:[\alpha, \beta] \times[a, b] \rightarrow \mathbb{R}$ we assume that

$$
\left\{\begin{array}{l}
u \mapsto f(k, u) \in C^{2}[a, b] \text { for all } k \in[\alpha, \beta] \\
k \mapsto f(k, u) \in C^{1}[\alpha, \beta] \text { for all } u \in[a, b]
\end{array}\right.
$$

Furthermore, we assume that $u \mapsto f(k(x, t), u)$ is genuinely nonlinear a.e. in $\mathbb{R} \times \mathbb{R}_{+}$. More precisely, for a.e. $(x, t) \in \mathbb{R} \times \mathbb{R}_{+}$, we assume

$$
f_{u u}(k(x, t), u) \neq 0 \quad \text { for a.e. } u \in[a, b] .
$$

Note that this also implies a condition on the coefficient $k(x, t)$. For example, if $f(k, u)$ has a multiplicative form $k f(u),(2.4)$ is satisfied if $f(u)$ is genuinely nonlinear (i.e., $f^{\prime \prime} \neq 0$ a.e.) and $|k(x, t)| \neq 0$ for a.e. $(x, t) \in \mathbb{R} \times \mathbb{R}_{+}$.

With the assumption (2.3) the partial derivatives $f_{k}$ and $f_{u}$ exist everywhere, and $\left\|f_{k}\right\|_{\infty}$ and $\left\|f_{u}\right\|_{\infty}$ denote the (finite) Lipschitz constants of $f_{k}$ and $f_{u}$ with respect to $(k, u) \in[\alpha, \beta] \times[a, b]$. With the notation $f_{k}$ and $f_{u}$, we always mean the first order partial derivatives of $f(\cdot, \cdot)$ with respect to the first and second variables.

We need also an assumption on $f$ that guarantees that the Lax-Friedrichs approximations stay uniformly bounded. For example, we can require

$$
f(k, a)=f(k, b)=0 \text { for all } k \in[\alpha, \beta],
$$

which in fact implies that the interval $[a, b]$ becomes an invariant region.

Next we describe the Lax-Friedrichs scheme. Let $\Delta x>0$ and $\Delta t>0$ denote the spatial and temporal discretization parameters, which are chosen so that they always obey the CFL condition

$$
\lambda\left\|f_{u}\right\|_{\infty} \leq 1-\kappa, \quad \lambda=\frac{\Delta t}{\Delta x}, \quad \text { for some } \kappa \in(0,1) .
$$

Here $\kappa$ is a positive parameter which we can choose to be very small so that the allowable time step is reduced only negligibly. We will work under the standing assumption that the space step $\Delta x$ and the time step $\Delta t$ are comparable, i.e., there are constants $c_{1}, c_{2}>0$ such that $c_{1} \leq \frac{\Delta t}{\Delta x} \leq c_{2}$.

The time domain $[0, \infty)$ is discretized via $t_{n}=n \Delta t$ for $n \in \mathbb{Z}_{+}^{0}:=\{0,1, \ldots\}\left(\mathbb{Z}_{+}:=\{1,2, \ldots\}\right)$, resulting in time strips $\left[t_{n}, t_{n+1}\right)$. The spatial domain $\mathbb{R}$ is divided into cells $\left[x_{j-1}, x_{j+1}\right)$ with centers at the points $x_{j}=j \Delta x$ for $j \in \mathbb{Z}$. Let $\chi_{j}(x)$ be the characteristic function for the interval $\left[x_{j-1}, x_{j+1}\right)$ and $\chi_{j}^{n}$ the characteristic function for the rectangle $\left[x_{j-1}, x_{j+1}\right) \times\left[t_{n}, t_{n+1}\right)$.

The finite difference scheme then generates, for each mesh size $\Delta=(\Delta x, \Delta t)$, with $\Delta x$ and $\Delta t$ taking values in sequences tending to zero, a piecewise constant approximation

$$
u^{\Delta}(x, t)=\sum_{n \in \mathbb{Z}_{+}^{0}} \sum_{\substack{j \in \mathbb{Z} \\ j+n=\text { even }}} \chi_{j}^{n}(x, t) U_{j}^{n},
$$

where the values $\left\{U_{j}^{n}:(j, n) \in \mathbb{Z} \times \mathbb{Z}_{+}^{0}, j+n=\right.$ even $\}$ remain to be defined. 
We define $\left\{U_{j}^{0}: j=\right.$ even $\}$ by

$$
U_{j}^{0}=\frac{1}{2 \Delta x} \int_{x_{j-1}}^{x_{j+1}} u_{0}(x) d x .
$$

Given $\left\{U_{j}^{n}: j+n=\right.$ even $\}$, we define next $\left\{U_{j}^{n+1}: j+n=\operatorname{odd}\right\}$. Let $(K, U)=(K, U)(x, t)$ denote a weak solution of the $2 \times 2$ system

$$
K_{t}=0, \quad U_{t}+f(K, U)_{x}=0, \quad(x, t) \in \mathbb{R} \times \mathbb{R}_{+},
$$

with Riemann initial data

$$
K(x, 0)=\left\{\begin{array}{ll}
k_{j-1}^{n}, & x<x_{j}, \\
k_{j+1}^{n}, & x>x_{j},
\end{array} \quad U(x, 0)= \begin{cases}U_{j-1}^{n}, & x<x_{j}, \\
U_{j+1}^{n}, & x>x_{j},\end{cases}\right.
$$

where the coefficient $k(x, t)$ has been discretized via the piecewise constant approximation

$$
k^{\Delta}(x, t)=\sum_{n \in \mathbb{Z}_{+}^{0}} \sum_{\substack{j \in \mathbb{Z} \\ j+n=\text { even }}} \chi_{j}^{n}(x, t) k_{j}^{n}, \quad k_{j}^{n}=\lim _{x \downarrow x_{j}} k\left(x, \hat{t}_{n}\right) .
$$

Here $\hat{t}_{n}$ is any point lying in the interval $\left[t_{n}, t_{n+1}\right)$ where the limit exists. With the assumption that $k \in B V_{\text {loc }}\left(\mathbb{R} \times \mathbb{R}_{+}\right)$, the limit in this formula exists for almost all $t \in\left[t_{n}, t_{n+1}\right)$, and so this definition makes sense. We then define

$$
U_{j}^{n+1}=\frac{1}{2 \Delta x} \int_{x_{j-1}}^{x_{j+1}} U(x, \Delta t) d x .
$$

Integrating the weak formulation of (2.9) over the control volume $\left[x_{j-1}, x_{j+1}\right) \times(0, \Delta t)$ gives

$$
\begin{aligned}
& \int_{x_{j-1}}^{x_{j+1}} U(x, \Delta t) d x=\int_{x_{j-1}}^{x_{j+1}} U(x, 0) d x \\
& \quad-\int_{0}^{\Delta t}\left(f\left(K\left(x_{j+1}, t\right), U\left(x_{j+1}, t\right)\right)-f\left(K\left(x_{j-1}, t\right), U\left(x_{j-1}, t\right)\right)\right) d t .
\end{aligned}
$$

After a direct evaluation of the integrals for $\Delta t$ small, we obtain the staggered Lax-Friedrichs scheme

$$
U_{j}^{n+1}=\frac{1}{2}\left(U_{j-1}^{n}+U_{j+1}^{n}\right)-\frac{\lambda}{2}\left(f\left(k_{j+1}^{n}, U_{j+1}^{n}\right)-f\left(k_{j-1}^{n}, U_{j-1}^{n}\right)\right),
$$

which also can be written in conservation form

$$
U_{j}^{n+1}=U_{j}^{n}-\lambda\left(\mathcal{F}_{j+1 / 2}^{n}-\mathcal{F}_{j-1 / 2}^{n}\right),
$$

where

$$
\mathcal{F}_{j+1 / 2}^{n}=\frac{1}{2}\left(f\left(k_{j}^{n}, U_{j}^{n}\right)+f\left(k_{j+1}^{n}, U_{j+1}^{n}\right)\right)-\frac{1}{2 \lambda} \Delta_{+} U_{j}^{n}
$$

is the Lax-Friedrichs numerical flux.

Notice that in this paper we restrict our attention to the sublattice

$$
\left\{\left(x_{j}, t_{n}\right): j+n=\text { even }\right\},
$$

which means that $\left\{U_{j}^{0}: j=\right.$ even $\},\left\{U_{j}^{1}: j=\right.$ odd $\},\left\{U_{j}^{2}: j=\right.$ even $\}$ etc. are calculated.

\section{A COMPENSATEd COMPACTNESS Lemma}

We provide a compensated compactness lemma $[23,24,25,30,31]$ that can be applied to scalar conservation laws with a space-time discontinuous flux. The compensated compactness method, and its applications to (systems of) conservation laws, is nicely reviewed in [4, 22].

Before we can prove the compensated compactness lemma, we need to recall the celebrated div-curl lemma. 
Lemma 3.1 (div-curl lemma). Let $\Omega \subset \mathbb{R}^{2}$ be an open domain. With $\varepsilon>0$ denoting a parameter taking its values in a sequence which tends to zero, suppose

Then along a subsequence

$$
\begin{aligned}
& D^{\varepsilon} \rightarrow D \quad \text { in }\left(L^{2}(\Omega)\right)^{2}, \quad E^{\varepsilon} \rightarrow E \quad \text { in }\left(L^{2}(\Omega)\right)^{2}, \\
& \left\{\operatorname{div} D^{\varepsilon}\right\}_{\varepsilon>0} \quad \text { lies in a compact subset of } W_{\mathrm{loc}}^{-1,2}(\Omega), \\
& \left\{\operatorname{curl} E^{\varepsilon}\right\}_{\varepsilon>0} \quad \text { lies in a compact subset of } W_{\mathrm{loc}}^{-1,2}(\Omega) .
\end{aligned}
$$

$$
D^{\varepsilon} \cdot E^{\varepsilon} \rightarrow D \cdot E \quad \text { in } \mathcal{D}^{\prime}(\Omega) .
$$

A feature of the proof of the compensated compactness lemma below is that it avoids the use of the Young measure by following an approach developed by Chen and $\mathrm{Lu}[4,22]$ for the standard scalar conservation law, i.e., (1.1) with $f=f(u)$. This is preferable as the fundamental theorem of Young measures applies most easily to functions that are continuous in all variables.

Lemma 3.2 (compensated compactness). Suppose the first part of (2.2), the first part of (2.3), and (2.4) hold. Suppose $\left\{u^{\varepsilon}\right\}_{\varepsilon>0}$ is a sequence of measurable functions on $\mathbb{R} \times \mathbb{R}_{+}$that satisfies the following two conditions:

(1) There exist two finite constants $a$ and $b$ with $a<b$, both independent of $\varepsilon$, such that

$$
a \leq u^{\varepsilon}(x, t) \leq b \quad \text { for a.e. }(x, t) \in \mathbb{R} \times \mathbb{R}_{+} .
$$

(2) The two sequences

$$
\begin{aligned}
& \left\{S_{1}\left(u^{\varepsilon}\right)_{t}+Q_{1}\left(k(x, t), u^{\varepsilon}\right)_{x}\right\}_{\varepsilon>0}, \\
& \left\{S_{2}\left(k(x, t), u^{\varepsilon}\right)_{t}+Q_{2}\left(k(x, t), u^{\varepsilon}\right)_{x}\right\}_{\varepsilon>0}
\end{aligned}
$$

belong to a compact subset of $W_{\mathrm{loc}}^{-1,2}\left(\mathbb{R} \times \mathbb{R}_{+}\right)$, where

$$
S_{1}(u)=u-c, \quad Q_{1}(k, u)=f(k, u)-f(k, c),
$$

and

$$
S_{2}(k, u)=f(k, u)-f(k, c), \quad Q_{2}(k, u)=\int_{c}^{u}\left(f_{u}(k, \xi)\right)^{2} d \xi,
$$

for any $c \in \mathbb{R}$.

Then there exists a subsequence of $\left\{u^{\varepsilon}\right\}_{\varepsilon>0}$ that converges a.e. to a function $u \in L^{\infty}\left(\mathbb{R} \times \mathbb{R}_{+}\right)$.

Proof. In what follows, we use "overline" to denote weak- $\star$ limits in $L^{\infty}\left(\mathbb{R} \times \mathbb{R}_{+}\right)$. Fix any bounded open set $\Omega \subset \mathbb{R} \times \mathbb{R}_{+}$, and introduce the vector fields

$$
D^{\varepsilon}=\left(S_{1}\left(u^{\varepsilon}\right), Q_{2}\left(k(x, t), u^{\varepsilon}\right)\right), \quad E^{\varepsilon}=\left(-Q_{2}\left(k(x, t), u^{\varepsilon}\right), S_{2}\left(k(x, t), u^{\varepsilon}\right)\right) .
$$

We can apply the div-curl lemma to the sequences $\left\{D^{\varepsilon}\right\}_{\varepsilon>0},\left\{E^{\varepsilon}\right\}_{\varepsilon>0}$. The result is the so-called Murat-Tartar commutator relation:

$$
\overline{D^{\varepsilon} \cdot E^{\varepsilon}}=\bar{D} \cdot \bar{E} \quad \text { a.e. in } \Omega,
$$

that is,

$$
\begin{aligned}
& \overline{\left(f^{\varepsilon}-\right.}f(k(x, t), c))^{2}-\left(u^{\varepsilon}-c\right) \int_{c}^{u^{\varepsilon}}\left(f_{u}(k(x, t), \xi)\right)^{2} d \xi \\
&=\left(\overline{\left(f^{\varepsilon}-f(k(x, t), c)\right)}\right)^{2}-\overline{\left(u^{\varepsilon}-c\right)} \overline{\int_{c}^{u^{\varepsilon}}\left(f_{u}(k(x, t), \xi)\right)^{2} d \xi}
\end{aligned}
$$

where $f^{\varepsilon}:=f\left(k(x, t), u^{\varepsilon}\right)$. We have

$$
\begin{aligned}
& \left(f^{\varepsilon}-f(k(x, t), c)\right)^{2} \\
& =\left(f^{\varepsilon}-f(k(x, t), \bar{u})\right)^{2}+2\left(f^{\varepsilon}-f(k(x, t), \bar{u})\right)(f(k(x, t), \bar{u})-f(k(x, t), c)) \\
& +(f(k(x, t), \bar{u})-f(k(x, t), c))^{2}
\end{aligned}
$$


and

$$
\begin{aligned}
& \left(u^{\varepsilon}-c\right) \int_{c}^{u^{\varepsilon}}\left(f_{u}(k(x, t), \xi)\right)^{2} d \xi \\
& =\left(u^{\varepsilon}-\bar{u}\right) \int_{\bar{u}}^{u^{\varepsilon}}\left(f_{u}(k(x, t), \xi)\right)^{2} d \xi+(\bar{u}-c) \int_{\bar{u}}^{u^{\varepsilon}}\left(f_{u}(k(x, t), \xi)\right)^{2} d \xi \\
& \quad+\left(u^{\varepsilon}-c\right) \int_{c}^{\bar{u}}\left(f_{u}(k(x, t), \xi)\right)^{2} d \xi .
\end{aligned}
$$

Using these identities in (3.2) we find that

$$
\begin{aligned}
& \left(f^{\varepsilon}-f(k(x, t), \bar{u})\right)^{2}-\left(u^{\varepsilon}-\bar{u}\right) \int_{\bar{u}}^{u^{\varepsilon}}\left(f_{u}(k(x, t), \xi)\right)^{2} d \xi \\
& +2 \overline{\left(f^{\varepsilon}-f(k(x, t), \bar{u})\right)}(f(k(x, t), \bar{u})-f(k(x, t), c)) \\
& +(f(k(x, t), \bar{u})-f(k(x, t), c))^{2}-(\bar{u}-c) \overline{\int_{\bar{u}}^{u^{\varepsilon}}\left(f_{u}(k(x, t), \xi)\right)^{2} d \xi} \\
& -\overline{\left(u^{\varepsilon}-c\right)} \int_{c}^{\bar{u}}\left(f_{u}(k(x, t), \xi)\right)^{2} d \xi \\
& =\left(\overline{\left(f^{\varepsilon}-f(k(x, t), c)\right)}\right)^{2}-\overline{\left(u^{\varepsilon}-c\right)} \overline{\int_{c}^{u^{\varepsilon}}\left(f_{u}(k(x, t), \xi)\right)^{2} d \xi}
\end{aligned}
$$

We have

$$
\begin{aligned}
& \left(\overline{\left(f^{\varepsilon}-f(k(x, t), c)\right)}\right)^{2} \\
& \quad=\left(\overline{\left(f^{\varepsilon}-f(k(x, t), \bar{u})\right)}\right)^{2} \\
& \quad 2 \overline{\left(f^{\varepsilon}-f(k(x, t), \bar{u})\right)}(f(k(x, t), \bar{u})-f(k(x, t), c)) \\
& \quad+(f(k(x, t), \bar{u})-f(k(x, t), c))^{2}
\end{aligned}
$$

and

$$
\begin{aligned}
& \overline{\left(u^{\varepsilon}-c\right)} \overline{\int_{c}^{u^{\varepsilon}} \frac{\left(f_{u}(k(x, t), \xi)\right)^{2} d \xi}{\overline{\int^{u^{\varepsilon}}}\left(f_{u}(k(x, t), \xi)\right)^{2} d \xi}}+(\bar{u}-c) \overline{\int_{\bar{u}}^{u^{\varepsilon}}\left(f_{u}(k(x, t), \xi)\right)^{2} d \xi} \\
& =\overline{\left(u^{\varepsilon}-\bar{u}\right.} \\
& \quad+\overline{\left(u^{\varepsilon}-c\right)} \int_{c}^{\bar{u}}\left(f_{u}(k(x, t), \xi)\right)^{2} d \xi \\
& =(\bar{u}-c) \overline{\int_{\bar{u}}^{u^{\varepsilon}}\left(f_{u}(k(x, t), \xi)\right)^{2} d \xi}+\overline{\left(u^{\varepsilon}-c\right)} \int_{c}^{\bar{u}}\left(f_{u}(k(x, t), \xi)\right)^{2} d \xi .
\end{aligned}
$$

Plugging these identities into (3.3) yields

$$
\overline{I\left(u^{\varepsilon}\right)}-\left(\overline{\left(f^{\varepsilon}-f(k(x, t), \bar{u})\right)}\right)^{2}=0 \quad \text { a.e. in } \Omega,
$$

where

$$
I\left(u^{\varepsilon}\right):=\left(f^{\varepsilon}-f(k(x, t), \bar{u})\right)^{2}-\left(u^{\varepsilon}-\bar{u}\right) \int_{\bar{u}}^{u^{\varepsilon}}\left(f_{u}(k(x, t), \xi)\right)^{2} d \xi .
$$

By the Cauchy-Schwartz inequality

$$
\begin{aligned}
\left(f^{\varepsilon}-f(k(x, t), \bar{u})\right)^{2} & =\left(\int_{\bar{u}}^{u^{\varepsilon}} f_{u}(k(x, t), \xi) d \xi\right)^{2} \\
& \leq\left(u^{\varepsilon}-u\right) \int_{\bar{u}}^{u^{\varepsilon}}\left(f_{u}(k(x, t), \xi)\right)^{2} d \xi
\end{aligned}
$$


Actually there is equality in (3.5) if and only if $f_{u u}(k(x, t), \xi)=0$ for all $\xi$ between $u$ and $u^{\varepsilon}$. This implies that both terms in (3.4) are nonpositive a.e. in $\Omega$, and thus they must be equal to zero. The second term being zero gives $\overline{f^{\varepsilon}}=f(k(x, t), \bar{u})$ for a.e. $(x, t) \in \Omega$. The first term in (3.4) being zero means that $\lim _{\varepsilon \downarrow 0} \iint_{\Omega} I\left(u^{\varepsilon}\right) \varphi(x, t) d x d t$ is zero for any function $\varphi \in L^{1}(\Omega)$. Hence $I\left(u^{\varepsilon}\right) \rightarrow 0$ a.e. in $\Omega$. In view of the "genuinely nonlinear" condition (2.4), and thus a strict inequality in (3.5), the nonpositive function $I(\cdot)$ has a strict global maximum at $\bar{u}$ with $I(\bar{u})=0$, so that

$$
I\left(u^{\varepsilon}\right) \leq-C_{\alpha} \text { a.e. on }\left\{\left|u^{\varepsilon}-\bar{u}\right|>\alpha\right\},
$$

for some constant $C_{\alpha}>0$ that depends on $\alpha$ but not $\varepsilon$. Consequently,

$$
\text { meas }\left\{\left|u^{\varepsilon}-\bar{u}\right|>\alpha\right\} \leq \frac{1}{C_{\alpha}} \underset{\Omega \cap\left|u^{\varepsilon}-\bar{u}\right|>\alpha}{\iint_{\varepsilon}} I_{\varepsilon}(x, t) d x d t \rightarrow 0 \quad \varepsilon \downarrow 0 .
$$

Since $\alpha>0$ was arbitrary, this shows that $u_{\varepsilon} \rightarrow \bar{u}$ in measure, which implies that a subsequence of $\left\{u^{\varepsilon}\right\}_{\varepsilon>0}$ converges to $\bar{u}$ a.e. in $\Omega$. Since $\Omega$ was arbitrary, a standard diagonal argument finishes the proof of the lemma.

Remark 3.3. By looking at Lemma 3.2 it becomes clear why we need to assume in (2.2) also some regularity in the time variable of $k(x, t)$.

When verifying (3.1) it is convenient to have following functional analysis lemma at our disposal $($ see $[4,22])$.

Lemma 3.4. Let $\Omega \subset \mathbb{R}^{d}$ be a bounded open set. Let $q$ and $r$ be a pair of constants satisfying $1<q \leq 2<r<\infty$. Then

$$
\begin{aligned}
& \left(\text { compact set of } W_{\mathrm{loc}}^{-1, q}(\Omega)\right) \cap\left(\text { bounded set of } W_{\mathrm{loc}}^{-1, r}(\Omega)\right) \\
& \subset\left(\text { compact set of } W_{\mathrm{loc}}^{-1,2}(\Omega)\right) .
\end{aligned}
$$

\section{Convergence analysis}

Now we set out to prove that the Lax-Friedrichs approximate solutions $\left\{u^{\Delta}\right\}_{\Delta>0}$ defined in Section 2 converge strongly to a weak solution of (1.1), at least along a subsequence. The general strategy of the convergence proof is in the spirit of the one used by DiPerna [7], and it has been used in various contexts and by many different authors since then (see, e.g., [4] for an overview). We first derive a uniform $L^{\infty}$ estimate via an invariant region principle. Then a precise entropy estimate is obtained for the quadratic entropy function $S(u)=\frac{1}{2} u^{2}$. The key point is that the entropy estimate is robust with respect to the smoothness of the coefficient $k(x, t)$. Moreover, it immediately provides us with discrete $L^{2}$ estimates on the spatial and temporal differences of the approximate solution $u^{\Delta}$, which imply the desired $W_{\text {loc }}^{-1,2}$ compactness of $\left\{u^{\Delta}\right\}_{\Delta>0}$. An application of the compensated compactness lemma from Section 3.2 then gives the desired strong convergence in $L^{p}$ for any $p<\infty$.

The first lemma guarantees that the scheme is monotone [6] (this will be used in Section 5 only) and that the approximations $U_{j}^{n}$ remain within the initial domain $[a, b]$.

Lemma 4.1 (monotonicity and $L^{\infty}$ estimate). Suppose the CFL condition (2.6) holds. Then the Lax-Friedrichs scheme (2.11) is monotone. Moreover, the computed approximations satisfy $u^{\Delta}(x, t) \in[a, b]$ for all $x$ and all $t \geq 0$.

Proof. Fix a time level $n \geq 0$, and for the sake of concreteness, assume that $n$ is odd. If $V_{j}^{n} \geq U_{j}^{n}$ for all odd $j$, then for $j$ even, the following relationship is easily derived from the definition (2.11) of the scheme:

$$
\begin{aligned}
V_{j}^{n+1}-U_{j}^{n+1}=\frac{1}{2} & \left(1-\lambda f_{u}\left(k_{j+1}^{n}, w_{j+1}^{n}\right)\right)\left(V_{j+1}^{n}-U_{j+1}^{n}\right) \\
& +\frac{1}{2}\left(1+\lambda f_{u}\left(k_{j-1}^{n}, w_{j-1}^{n}\right)\right)\left(V_{j-1}^{n}-U_{j-1}^{n}\right),
\end{aligned}
$$


where $w_{j-1}^{n} \in \operatorname{co}\left(U_{j-1}^{n}, V_{j-1}^{n}\right)$ and $w_{j+1}^{n} \in \operatorname{co}\left(U_{j+1}^{n}, V_{j+1}^{n}\right)$. It is clear that the right side of (4.1) is nonnegative if the CFL condition (2.6) is satisfied, which proves that the scheme is monotone. To demonstrate the invariance of the initial domain $[a, b]$, we start with the observation that the functions $w^{\Delta}(x, t) \equiv a, v^{\Delta}(x, t) \equiv b$ are fixed point solutions of the finite difference equation (2.11). This is a result of assumption (2.5). At the initial time level,

$$
a=w^{\Delta}\left(x, t_{0}\right) \leq u^{\Delta}\left(x, t_{0}\right) \leq v^{\Delta}\left(x, t_{0}\right)=b,
$$

and thus monotonicity, along with the fixed point property of $w^{\Delta}(x, t)$ and $v^{\Delta}(x, t)$, yields

$$
a=w^{\Delta}\left(x, t_{1}\right) \leq u^{\Delta}\left(x, t_{1}\right) \leq v^{\Delta}\left(x, t_{1}\right)=b .
$$

Clearly we can continue this way inductively, showing that the computed approximations satisfy $a \leq u^{\Delta}\left(x, t^{n}\right) \leq b$ for all $n \geq 0$.

Lax [19] derived an entropy estimate for the Lax-Friedrichs scheme as it applies to a hyperbolic system of conservation laws admitting a strictly convex entropy. The following lemma, as well as its proof, is an adaptation of that entropy estimate to the context of this paper.

Lemma 4.2 (entropy estimate). Let $(S, Q)$ be defined by

$$
S(u)=\frac{1}{2} u^{2}, \quad Q_{u}(k, u)=u f_{u}(k, u) .
$$

With $k_{j-1}^{n}, U_{j-1}^{n}$ and $k_{j+1}^{n}, U_{j+1}^{n}$ given, compute $U_{j}^{n+1}$ by (2.11). Then

$$
\begin{aligned}
& S\left(U_{j}^{n+1}\right)-\frac{1}{2}\left(S\left(U_{j-1}^{n}\right)+S\left(U_{j+1}^{n}\right)\right)+\frac{\lambda}{2}\left(Q\left(k_{j+1}^{n}, U_{j+1}^{n}\right)-Q\left(k_{j-1}^{n}, U_{j-1}^{n}\right)\right) \\
& \leq-\frac{\kappa^{2}}{8}\left(U_{j+1}^{n}-U_{j-1}^{n}\right)^{2}+\mathcal{O}\left(\left|k_{j+1}^{n}-k_{j-1}^{n}\right|\right) .
\end{aligned}
$$

Proof. Let us introduce the functions $w, v, \Phi:[a, b] \rightarrow \mathbb{R}$ defined by

$$
\begin{aligned}
w(s) & =s U_{j-1}^{n}+(1-s) U_{j+1}^{n}, \\
v(s) & =\frac{1}{2}\left(w(s)+U_{j+1}^{n}\right)-\frac{\lambda}{2}\left(f\left(k_{j+1}^{n}, U_{j+1}^{n}\right)-f\left(k_{j-1}^{n}, w(s)\right)\right), \\
\Phi(s) & =\frac{1}{2}\left(S(w(s))+S\left(U_{j+1}^{n}\right)\right)+\frac{\lambda}{2}\left(Q\left(k_{j-1}^{n}, w(s)\right)-Q\left(k_{j+1}^{n}, U_{j+1}^{n}\right)\right)-S(v(s)) .
\end{aligned}
$$

It will be useful to have the following elementary facts about these functions collected in one place before continuing with the proof:

$$
\begin{aligned}
& w(0)=U_{j+1}^{n}, \quad w(1)=U_{j-1}^{n}, \quad w^{\prime}(s)=U_{j-1}^{n}-U_{j+1}^{n}, \\
& v(0)=U_{j+1}^{n}-\frac{\lambda}{2}\left(f\left(k_{j+1}^{n}, U_{j+1}^{n}\right)-f\left(k_{j-1}^{n}, U_{j+1}^{n}\right)\right), \quad v(1)=U_{j}^{n+1}, \\
& v^{\prime}(s)=\frac{1}{2}\left(1+\lambda f_{u}\left(k_{j-1}^{n}, w(s)\right)\right) w^{\prime}(s)=\frac{1}{2}\left(1+\lambda f_{u}\left(k_{j-1}^{n}, w(s)\right)\right)\left(U_{j-1}^{n}-U_{j+1}^{n}\right), \\
& \Phi(0)=S\left(U_{j+1}^{n}\right)-\frac{\lambda}{2}\left(Q\left(k_{j+1}^{n}, U_{j+1}^{n}\right)-Q\left(k_{j-1}^{n}, U_{j+1}^{n}\right)\right) \\
& \quad-S\left(U_{j+1}^{n}-\frac{\lambda}{2}\left(f\left(k_{j+1}^{n}, U_{j+1}^{n}\right)-f\left(k_{j-1}^{n}, U_{j+1}^{n}\right)\right)\right), \\
& \Phi(1)=\frac{1}{2}\left(S\left(U_{j-1}^{n}\right)+S\left(U_{j+1}^{n}\right)\right) \\
& \quad+\frac{\lambda}{2}\left(Q\left(k_{j-1}^{n}, U_{j-1}^{n}\right)-Q\left(k_{j+1}^{n}, U_{j+1}^{n}\right)\right)-S\left(U_{j}^{n+1}\right) .
\end{aligned}
$$

Now the main point is to estimate $\Phi^{\prime}(s)$. Straightforward calculations show that

$$
\Phi^{\prime}(s)=\frac{1}{2}\left(1+\lambda f_{u}\left(k_{j-1}^{n}, w(s)\right)\right)\left(U_{j-1}^{n}-U_{j+1}^{n}\right)(w(s)-v(s))
$$


and

$$
\begin{aligned}
w(s)-v(s)=\frac{1}{2}(1- & \left.\lambda \frac{f\left(k_{j-1}^{n}, w(s)\right)-f\left(k_{j-1}^{n}, U_{j+1}^{n}\right)}{w(s)-U_{j+1}^{n}}\right)\left(w(s)-U_{j+1}^{n}\right) \\
& +\frac{\lambda}{2}\left(f\left(k_{j+1}^{n}, U_{j+1}\right)-f\left(k_{j-1}^{n}, U_{j+1}\right)\right)
\end{aligned}
$$

so that, with $A:=1-\lambda\left(f\left(k_{j-1}^{n}, w(s)\right)-f\left(k_{j-1}^{n}, U_{j+1}^{n}\right)\right) /\left(w(s)-U_{j+1}^{n}\right)$,

$$
\begin{aligned}
w(s)-v(s) & =\frac{A}{2}\left(w(s)-U_{j+1}^{n}\right)+\frac{\lambda}{2}\left(f\left(k_{j+1}^{n}, U_{j+1}^{n}\right)-f\left(k_{j-1}^{n}, U_{j+1}^{n}\right)\right) \\
& =\frac{A}{2}\left(U_{j-1}^{n}-U_{j+1}^{n}\right) s+\frac{\lambda}{2}\left(f\left(k_{j+1}^{n}, U_{j+1}^{n}\right)-f\left(k_{j-1}^{n}, U_{j+1}^{n}\right)\right) .
\end{aligned}
$$

As a consequence of the CFL condition (2.6), $A \geq \kappa$. Similarly, the quantity $1+\lambda f_{u}\left(k_{j-1}^{n}, w(s)\right)$ appearing in (4.3) is not less than $\kappa$. Thus,

$$
\Phi^{\prime}(s) \geq \frac{\kappa^{2}}{4}\left(U_{j-1}^{n}-U_{j+1}^{n}\right)^{2} s-C\left|k_{j+1}^{n}-k_{j-1}^{n}\right|,
$$

for some positive constant $C$ independent of $\Delta$.

Integrating this last inequality from 0 to 1 gives

$$
\Phi(1)-\Phi(0) \geq \frac{\kappa^{2}}{8}\left(U_{j-1}^{n}-U_{j+1}^{n}\right)^{2}-C\left|k_{j+1}^{n}-k_{j-1}^{n}\right|,
$$

which concludes the proof of the lemma as soon as we show that $-\Phi(0)$ is bounded by a constant times $\left|k_{j+1}^{n}-k_{j-1}^{n}\right|$. Using convexity of $S$,

$$
\begin{aligned}
& S\left(U_{j+1}^{n}-\frac{\lambda}{2}\left(f\left(k_{j+1}^{n}, U_{j+1}^{n}\right)-f\left(k_{j-1}^{n}, U_{j+1}^{n}\right)\right)\right) \\
& \quad \leq S\left(U_{j+1}^{n}\right)-S^{\prime}\left(U_{j+1}^{n}\right) \frac{\lambda}{2}\left(f\left(k_{j+1}^{n}, U_{j+1}^{n}\right)-f\left(k_{j-1}^{n}, U_{j+1}^{n}\right)\right),
\end{aligned}
$$

and inserting this estimate into $-\Phi(0)$ yields easily the desired upper bound.

The next lemma is a consequence of the entropy estimate.

Lemma 4.3 ( $L^{2}$ estimates on spatial/temporal differences). For $T>0, N=\lfloor T / \Delta t\rfloor$, and $X>0$, $J=\lfloor X / 2 \Delta x\rfloor+2$ we have the bounds

$$
\begin{aligned}
& \Delta x \sum_{n=0}^{N} \sum_{\substack{|j| \leq J \\
j+n+1=\text { even }}}\left(U_{j+1}^{n}-U_{j-1}^{n}\right)^{2} \leq C_{1}(X, T)<\infty, \\
& \sum_{n=0}^{N-1} \int_{-X}^{X}\left(u^{\Delta}\left(x, t_{n+1}\right)-u^{\Delta}\left(x, t_{n}\right)\right)^{2} d x \leq C_{2}(X, T)<\infty,
\end{aligned}
$$

where $C_{1}(X, T)$ and $C_{2}(X, T)$ are independent of $\Delta$. 
Proof. Starting from (4.2), we derive the following estimate by exploiting the telescoping nature of the sum and taking into account the boundary terms.

$$
\begin{aligned}
& \frac{\kappa^{2} \Delta x}{8} \sum_{n=0}^{N} \sum_{\substack{|j| \leq J \\
j+n+1=\text { even }}}\left(U_{j+1}^{n}-U_{j-1}^{n}\right)^{2} \\
& \leq \frac{\Delta x}{2} \sum_{|j| \leq J}\left(U_{j}^{0}\right)^{2}+\frac{\Delta t}{2} \sum_{n=0}^{N}\left(\left|Q\left(k_{J-1}^{n}, U_{J-1}^{n}\right)\right|+\left|Q\left(k_{J+1}^{n}, U_{J+1}^{n}\right)\right|\right) \\
& \quad+\frac{\Delta t}{2 \lambda} \sum_{n=0}^{N}\left(\left|S\left(U_{J-1}^{n}\right)\right|+\left|S\left(U_{J+1}^{n}\right)\right|\right)+\mathcal{O}\left(\Delta x \sum_{n=0}^{N} \sum_{\substack{j \in \mathbb{Z} \\
j+n=\text { even }}}\left|k_{j+2}^{n}-k_{j}^{n}\right|\right)
\end{aligned}
$$

The first part of (4.5) now clearly follows from (2.1) and (2.2), as well as the fact that $S$ and $Q$ are continuous.

To prove the second part of (4.5), fix $n \geq 0$. Then, taking into account the staggered nature of the mesh and our choice of $J$,

$$
\begin{aligned}
\int_{-X}^{X} & \left(u^{\Delta}\left(x, t^{n+1}\right)-u^{\Delta}\left(x, t^{n}\right)\right)^{2} d x \\
& \leq \Delta x \sum_{\substack{|j| \leq J \\
j+n+1=\text { even }}}\left(\left(U_{j}^{n+1}-U_{j-1}^{n}\right)^{2}+\left(U_{j}^{n+1}-U_{j+1}^{n}\right)^{2}\right) .
\end{aligned}
$$

Using the definition of the difference algorithm, and then Jensen's inequality, we get

$$
\begin{aligned}
\left(U_{j}^{n+1}-U_{j-1}^{n}\right)^{2} & =\left(\frac{1}{2}\left(U_{j+1}^{n}-U_{j-1}^{n}\right)-\frac{1}{2} \lambda\left(f\left(k_{j+1}^{n}, U_{j+1}^{n}\right)-f\left(k_{j-1}^{n}, U_{j-1}^{n}\right)\right)\right)^{2} \\
& \leq \frac{1}{2}\left(U_{j+1}^{n}-U_{j-1}^{n}\right)^{2}+\frac{1}{2}\left(\lambda\left(f\left(k_{j+1}^{n}, U_{j+1}^{n}\right)-f\left(k_{j-1}^{n}, U_{j-1}^{n}\right)\right)\right)^{2} .
\end{aligned}
$$

An application of the inequality $(a+b)^{2} \leq \frac{1}{2} a^{2}+\frac{1}{2} b^{2}$ yields

$$
\begin{aligned}
& \left(f\left(k_{j+1}^{n}, U_{j+1}^{n}\right)-f\left(k_{j-1}^{n}, U_{j-1}^{n}\right)\right)^{2} \\
& \quad \leq \frac{1}{2}\left\|f_{u}\right\|_{\infty}^{2}\left(U_{j+1}^{n}-U_{j-1}^{n}\right)^{2}+\frac{1}{2}\left\|f_{k}\right\|_{\infty}^{2}\left(k_{j+1}^{n}-k_{j-1}^{n}\right)^{2} \\
& \quad \leq \frac{1}{2}\left\|f_{u}\right\|_{\infty}^{2}\left(U_{j+1}^{n}-U_{j-1}^{n}\right)^{2}+\left\|f_{k}\right\|_{\infty}^{2}\|k\|_{L^{\infty}(\mathbb{R})}\left|k_{j+1}^{n}-k_{j-1}^{n}\right| .
\end{aligned}
$$

Using this estimate in (4.8) gives

$$
\begin{aligned}
\left(U_{j}^{n+1}-U_{j-1}^{n}\right)^{2} \leq \frac{1}{2}(1 & \left.+\frac{1}{2} \lambda^{2}\left\|f_{u}\right\|_{\infty}^{2}\right)\left(U_{j+1}^{n}-U_{j-1}^{n}\right)^{2} \\
& +\frac{1}{2} \lambda^{2}\left\|f_{k}\right\|_{\infty}^{2}\|k\|_{L^{\infty}(\mathbb{R})}\left|k_{j+1}^{n}-k_{j-1}^{n}\right| .
\end{aligned}
$$

A similar calculation provides the same estimate for the quantity $\left(U_{j}^{n+1}-U_{j+1}^{n}\right)^{2}$. Substituting these estimates into (4.7), the result is

$$
\begin{aligned}
& \int_{-X}^{X}\left(u^{\Delta}\left(x, t^{n+1}\right)-u^{\Delta}\left(x, t^{n}\right)\right)^{2} d x \\
& \leq 2 \Delta x \sum_{\substack{|j| \leq J \\
j+n+1=\text { even }}}\left(\left(1+\frac{1}{2} \lambda^{2}\left\|f_{u}\right\|_{\infty}^{2}\right)\left(U_{j+1}^{n}-U_{j-1}^{n}\right)^{2}\right. \\
& \\
&\left.\quad+\lambda^{2}\left\|f_{k}\right\|_{\infty}^{2}\|k\|_{L^{\infty}(\mathbb{R})}\left|k_{j+1}^{n}-k_{j-1}^{n}\right|\right) .
\end{aligned}
$$


The second part of (4.5) now follows by summing (4.10) over $n \in\{0, \ldots, N-1\}$, and invoking the first part of (4.5), along with the assumption that $k(x, t)$ belongs to $B V_{\text {loc }}\left(\mathbb{R} \times \mathbb{R}_{+}\right)$.

Now we prove the $W_{\text {loc }}^{-1,2}$ compactness of the Lax-Friedrichs approximate solutions.

Lemma $4.4\left(W_{\text {loc }}^{-1,2}\right.$ compactness). For any function $S(k, u)$ having the same regularity as $f(k, u)$, the sequence of distributions

$$
\left\{S\left(k(x, t), u^{\Delta}\right)_{t}+Q\left(k(x, t), u^{\Delta}\right)_{x}\right\}_{\Delta>0}
$$

lies in a compact subset of $W_{\mathrm{loc}}^{-1,2}\left(\mathbb{R} \times \mathbb{R}_{+}\right)$, where $Q_{u}(k, u)=S_{u}(k, u) f_{u}(k, u)$.

Proof. Let $\phi \in \mathcal{D}([-X, X] \times[0, T])$ for some $X>0, T>0$, and fix indices $N:=\lfloor T / \Delta t]$, $J=\lfloor X / 2 \Delta x\rfloor+1$. Define

$$
\left\langle\mathcal{L}^{\Delta}, \phi\right\rangle=\int_{\mathbb{R}_{+}} \int_{\mathbb{R}}\left(S\left(k(x, t), u^{\Delta}\right) \phi_{t}+Q\left(k(x, t), u^{\Delta}\right) \phi_{x}\right) d x d t .
$$

Let us first write $\mathcal{L}^{\Delta}=\mathcal{L}_{1}^{\Delta}+\mathcal{L}_{2}^{\Delta}$, where

$$
\begin{aligned}
\left\langle\mathcal{L}_{1}^{\Delta}, \phi\right\rangle= & \int_{\mathbb{R}_{+}} \int_{\mathbb{R}}\left(S\left(k(x, t), u^{\Delta}\right)-S\left(k^{\Delta}(x, t), u^{\Delta}\right)\right) \phi_{t} d x d t \\
& \quad+\int_{\mathbb{R}_{+}} \int_{\mathbb{R}}\left(Q\left(k(x, t), u^{\Delta}\right)-Q\left(k^{\Delta}(x, t), u^{\Delta}\right)\right) \phi_{x} d x d t \\
\left\langle\mathcal{L}_{2}^{\Delta}, \phi\right\rangle= & \int_{\mathbb{R}_{+}} \int_{\mathbb{R}}\left(S\left(k^{\Delta}(x, t), u^{\Delta}\right) \phi_{t}+Q\left(k^{\Delta}(x, t), u^{\Delta}\right) \phi_{x}\right) d x d t
\end{aligned}
$$

In what follows, we let $\Omega$ denote an arbitrary but fixed bounded open subset of $\mathbb{R} \times \mathbb{R}_{+}$. Let $q_{1} \in(1,2]$ and set $p=\frac{q_{1}}{q_{1}-1} \in[1, \infty)$. With $\phi \in W_{0}^{1, q_{1}}(\Omega)$, we have by Hölder's inequality

$$
\left|\left\langle\mathcal{L}_{1}^{\Delta}, \phi\right\rangle\right| \leq C\left\|k-k^{\Delta}\right\|_{L^{p}(\Omega)}\|\phi\|_{W_{0}^{1, q_{1}}(\Omega)} \rightarrow 0 \quad \text { as } \Delta \downarrow 0,
$$

so that

$$
\left\{\mathcal{L}_{1}^{\Delta}\right\}_{\Delta>0} \quad \text { is compact in } \quad W^{-1, q_{1}}(\Omega), \quad q_{1} \in(1,2] .
$$

In what follows, we employ the simplifying notation

$$
S_{j}^{n}:=S\left(k^{\Delta}\left(x_{j}, t_{n}\right), u^{\Delta}\left(x_{j}, t_{n}\right)\right), \quad Q_{j}^{n}:=Q\left(k^{\Delta}\left(x_{j}, t_{n}\right), u^{\Delta}\left(x_{j}, t_{n}\right)\right),
$$


and similarly for $\phi_{j}^{n}$. We decompose $\mathcal{L}_{2}^{\Delta}$ as follows:

$$
\begin{aligned}
&\left\langle\mathcal{L}_{2}^{\Delta}, \phi\right\rangle= \sum_{n=0}^{N-1} \sum_{\substack{j \in \mathbb{Z} \\
j+n=\text { even }}} \int_{t_{n}}^{t_{n+1}} \int_{x_{j-1}}^{x_{j+1}}\left(S\left(k^{\Delta}, u^{\Delta}\right) \phi_{t}+Q\left(k^{\Delta}, u^{\Delta}\right) \phi_{x}\right) d x d t \\
&= \sum_{n=0}^{N-1} \sum_{\substack{j \in \mathbb{Z} \\
j+n=\text { even }}} \int_{x_{j-1}}^{x_{j+1}} S\left(k^{\Delta}, u^{\Delta}\right)\left(\phi\left(x, t_{n+1}\right)-\phi\left(x, t_{n}\right)\right) d x \\
&+\sum_{n=0}^{N-1} \sum_{\substack{j \in \mathbb{Z} \\
j+n=\text { even }}} \int_{t_{n}}^{t_{n+1}} Q\left(k^{\Delta}, u^{\Delta}\right)\left(\phi\left(x_{j+1}, t\right)-\phi\left(x_{j-1}, t\right)\right) d t \\
&= \sum_{j=\text { even }} \int_{x_{j-1}}^{x_{j+1}} S_{j}^{N} \phi(x, N \Delta t) d x-\sum_{j=\text { even }} \int_{x_{j-1}}^{x_{j+1}} S_{j}^{0} \phi(x, 0) d x \\
&-\sum_{n=1}^{N} \sum_{\substack{j \in \mathbb{Z} \\
j+n=\text { even }}} \int_{x_{j-1}}^{x_{j}}\left(S_{j}^{n}-S_{j-1}^{n-1}\right) \phi\left(x, t_{n}\right) d x \\
&-\sum_{n=1}^{N} \sum_{\substack{j \in \mathbb{Z} \\
j+n=\text { even }}} \int_{x_{j}}^{x_{j+1}}\left(S_{j}^{n}-S_{j+1}^{n-1}\right) \phi\left(x, t_{n}\right) d x \\
&=\left\langle\sum_{n=1}^{N} \sum_{\substack{j \in \mathbb{Z} \\
j+n=\text { even }}} \int_{t_{n-1}}^{t_{n}}\left(Q_{j+1}^{n-1}-\phi\right\rangle+\left\langle Q_{j-1}^{n-1}\right) \phi\left(x_{j}, t\right) d t\right. \\
&\left.+\mathcal{L}_{2,1}^{\Delta}, \phi\right\rangle+\left\langle\mathcal{L}_{2,2}^{\Delta}, \phi\right\rangle+\left\langle\mathcal{L}_{2,3}^{\Delta}, \phi\right\rangle+\left\langle\mathcal{L}_{2,4}^{\Delta}, \phi\right\rangle,
\end{aligned}
$$

where (after some work)

$$
\begin{aligned}
\left\langle\mathcal{L}_{2,0}^{\Delta}, \phi\right\rangle & =\sum_{j=\mathrm{even}} \int_{x_{j-1}}^{x_{j+1}} S_{j}^{N} \phi(x, N \Delta t) d x-\sum_{j=\mathrm{even}} \int_{x_{j-1}}^{x_{j+1}} S_{j}^{0} \phi(x, 0) d x \\
\left\langle\mathcal{L}_{2,1}^{\Delta}, \phi\right\rangle & =-2 \Delta x \sum_{n=1}^{N} \sum_{\substack{j \in \mathbb{Z} \\
j+n=\mathrm{even}}}\left\{\left(S_{j}^{n}-\frac{1}{2} S_{j-1}^{n-1}-\frac{1}{2} S_{j+1}^{n-1}\right)+\frac{\lambda}{2}\left(Q_{j+1}^{n-1}-Q_{j-1}^{n-1}\right)\right\} \phi_{j}^{n} \\
\left\langle\mathcal{L}_{2,2}^{\Delta}, \phi\right\rangle & =\sum_{n=1}^{N} \sum_{\substack{j \in \mathbb{Z} \\
j+n=\mathrm{even}}} \int_{x_{j-1}}^{x_{j}}\left(S_{j-1}^{n-1}-S_{j}^{n}\right)\left(\phi_{j}^{n}-\phi\left(x, t_{n}\right)\right) d x, \\
\left\langle\mathcal{L}_{2,3}^{\Delta}, \phi\right\rangle & =\sum_{n=1}^{N} \sum_{\substack{j \in \mathbb{Z} \\
j+n=\text { even }}} \int_{x_{j}}^{x_{j+1}}\left(S_{j+1}^{n-1}-S_{j}^{n}\right)\left(\phi_{j}^{n}-\phi\left(x, t_{n}\right)\right) d x, \\
\left\langle\mathcal{L}_{2,4}^{\Delta}, \phi\right\rangle & =\sum_{n=1}^{N} \sum_{\substack{j \in \mathbb{Z} \\
j+n=\mathrm{even}}} \int_{t_{n-1}}^{t_{n}}\left(Q_{j+1}^{n-1}-Q_{j-1}^{n-1}\right)\left(\phi_{j}^{n}-\phi\left(x_{j}, t\right)\right) d t .
\end{aligned}
$$


For the term $\left\langle\mathcal{L}_{2,1}^{\Delta}, \phi\right\rangle$, we claim that

$$
\begin{aligned}
& \left|\left\langle\mathcal{L}_{2,1}^{\Delta}, \phi\right\rangle\right| \\
& =\left|-2 \Delta x \sum_{n=1}^{N} \sum_{\substack{j \in \mathbb{Z} \\
j+n=\text { even }}}\left\{\left(S_{j}^{n}-\frac{1}{2} S_{j-1}^{n-1}-\frac{1}{2} S_{j+1}^{n-1}\right)+\frac{\lambda}{2}\left(Q_{j+1}^{n-1}-Q_{j-1}^{n-1}\right)\right\} \phi_{j}^{n}\right| \\
& =\mathcal{O}\left(\Delta x \sum_{n=1}^{N} \sum_{\substack{|j| \leq J \\
j+n+1=\text { even }}}\left(U_{j+1}^{n-1}-U_{j-1}^{n-1}\right)^{2}+|k|_{B V([-X, X] \times[0, T])}\right)\|\phi\|_{L^{\infty}(\Omega)} .
\end{aligned}
$$

To prove this claim, we first observe that in the expression

$$
\left(S_{j}^{n}-\frac{1}{2} S_{j-1}^{n-1}-\frac{1}{2} S_{j+1}^{n-1}\right)+\frac{\lambda}{2}\left(Q_{j+1}^{n-1}-Q_{j-1}^{n-1}\right)
$$

we may replace $S_{j \pm 1}^{n-1}=S\left(k^{\Delta}\left(x_{j \pm 1}, t_{n-1}\right), u^{\Delta}\left(x_{j \pm 1}, t_{n-1}\right)\right)$ by

$$
S\left(k^{\Delta}\left(x_{j}, t_{n}\right), u^{\Delta}\left(x_{j \pm 1}, t_{n-1}\right)\right):=\widehat{S}_{j \pm 1}^{n-1},
$$

and absorb the difference in the " $|k|_{B V}$ term". With this simplification, we can follow the first part of the proof of Lemma 4.2, with $S\left(k^{\Delta}\left(x_{j}, t_{n}\right), u\right)$ playing the role of $S(u)$, noting that we must replace the formula (4.3) for $\Phi^{\prime}(s)$ by

$$
\Phi^{\prime}(s)=\frac{1}{2}\left(1+\lambda f_{u}\left(k_{j-1}^{n}, w(s)\right)\right)\left(U_{j-1}^{n}-U_{j+1}^{n}\right) S^{\prime \prime}(\theta)(w(s)-v(s)),
$$

where $\theta$ lies between $w(s)$ and $v(s)$. Now for $s \in[0,1]$, both $w(s)$ and $v(s)$ lie in $[a, b]$, giving us a uniform bound on $S^{\prime \prime}(\theta)$ :

$$
\left|S^{\prime \prime}(\theta)\right| \leq \max _{w \in[a, b]}\left|S^{\prime \prime}(w)\right|:=B .
$$

We then find that

$$
\begin{aligned}
& \left|\left(S_{j}^{n}-\frac{1}{2} \widehat{S}_{j-1}^{n-1}-\frac{1}{2} \widehat{S}_{j+1}^{n-1}\right)+\frac{\lambda}{2}\left(Q_{j+1}^{n-1}-Q_{j-1}^{n-1}\right)\right| \\
& \quad=|\Phi(1)-\Phi(0)|+\mathcal{O}\left(\left|k_{j+1}^{n}-k_{j-1}^{n}\right|\right) \leq \max _{s \in[0,1]}\left|\Phi^{\prime}(s)\right|+\mathcal{O}\left(\left|k_{j+1}^{n}-k_{j-1}^{n}\right|\right) .
\end{aligned}
$$

Referring to (4.14) and (4.4), and recalling the CFL condition 2.6, we find that for $s \in[0,1]$

$$
\begin{aligned}
\left|\Phi^{\prime}(s)\right| & \leq B\left|U_{j+1}^{n}-U_{j-1}^{n}\right||w(s)-v(s)| \\
& \leq B\left|U_{j+1}^{n}-U_{j-1}^{n}\right|\left(\frac{1}{2}\left|U_{j+1}^{n}-U_{j-1}^{n}\right|+\frac{\lambda}{2}\left\|f_{k}\right\|_{\infty}\left|k_{j+1}^{n}-k_{j-1}^{n}\right|\right) \\
& \leq \frac{B}{2}\left(U_{j+1}^{n}-U_{j-1}^{n}\right)^{2}+\frac{B \lambda}{2}\left\|f_{k}\right\|_{\infty}\left|U_{j+1}^{n}-U_{j-1}^{n}\right|\left|k_{j+1}^{n}-k_{j-1}^{n}\right| \\
& \leq B\left(\frac{1}{2}+\frac{\lambda}{4}\left\|f_{k}\right\|_{\infty}\right)\left(U_{j+1}^{n}-U_{j-1}^{n}\right)^{2}+\frac{B \lambda}{4}\left\|f_{k}\right\|_{\infty}\left(k_{j+1}^{n}-k_{j-1}^{n}\right)^{2} \\
& \leq B\left(\frac{1}{2}+\frac{\lambda}{4}\left\|f_{k}\right\|_{\infty}\right)\left(U_{j+1}^{n}-U_{j-1}^{n}\right)^{2}+\frac{B \lambda}{2}\left\|f_{k}\right\|_{\infty}\|k\|_{\infty}\left|k_{j+1}^{n}-k_{j-1}^{n}\right| .
\end{aligned}
$$

Substituting this estimate into (4.15), it is now clear that the claim (4.12) holds.

Now by combining the estimate (4.12) with the first part of (4.5) and and (2.2), we see that

$$
\left|\left\langle\mathcal{L}_{2,1}^{\Delta}, \phi\right\rangle\right| \leq C\|\phi\|_{L^{\infty}(\Omega)}, \quad \phi \in C_{0}(\Omega),
$$

which yields the uniform bound

$$
\left\|\mathcal{L}_{2,0}^{\Delta}\right\|_{\mathcal{M}(\Omega)},\left\|\mathcal{L}_{2,1}^{\Delta}\right\|_{\mathcal{M}(\Omega)} \leq C
$$


where $\mathcal{M}(\Omega)=\left(C_{c}(\Omega)\right)^{\star}$ denotes the space of bounded measures on $\Omega$. Sobolev's imbedding theorem gives $\mathcal{M}(\Omega) \subset W^{-1, q_{2}}(\Omega)$ with compact injection for any $q_{2} \in(1,2)$. Hence

$$
\left\{\mathcal{L}_{2,1}^{\Delta}\right\}_{\Delta>0} \quad \text { is compact in } \quad W^{-1, q_{2}}(\Omega), \quad q_{2} \in(1,2) .
$$

To estimate $\left\langle\mathcal{L}_{2,2}^{\Delta}, \phi\right\rangle$, we use Hölder's inequality to get

$$
\begin{aligned}
\left|\left\langle\mathcal{L}_{2,2}^{\Delta}, \phi\right\rangle\right| \leq \Delta x^{-\frac{1}{2}}\{\Delta & \left.x \sum_{n=1}^{N} \sum_{\substack{|j| \leq J \\
j+n=\text { even }}}\left(S_{j-1}^{n-1}-S_{j}^{n}\right)^{2}\right\}^{\frac{1}{2}} \\
& \times\left\{\sum_{n=1}^{N} \sum_{\substack{|j| \leq J \\
j+n=\text { even }}}\left(\int_{x_{j-1}}^{x_{j}}\left(\phi_{j}^{n}-\phi\left(x, t^{n}\right)\right) d x\right)^{2}\right\}^{\frac{1}{2}} .
\end{aligned}
$$

Using (4.5), see also (4.7), and (2.2), we obtain

$$
\begin{aligned}
& \Delta x \sum_{n=1}^{N} \sum_{\substack{|j| \leq J \\
j+n=\text { even } \\
j+1}}\left(S_{j-1}^{n-1}-S_{j}^{n}\right)^{2} \\
& \quad \leq C \Delta x \sum_{n=1}^{N} \sum_{\substack{|j| \leq J \\
j+n=\text { even }}}\left\{\left(U_{j}^{n}-U_{j-1}^{n-1}\right)^{2}+\left(k_{j}^{n}-k_{j-1}^{n-1}\right)^{2}\right\} \leq C,
\end{aligned}
$$

so that

$$
\left|\left\langle\mathcal{L}_{2,2}^{\Delta}, \phi\right\rangle\right| \leq C\|\phi\|_{C_{0}^{\alpha}(\Omega)} \Delta x^{\alpha-\frac{1}{2}}, \quad \phi \in C_{0}^{\alpha}(\Omega), \alpha \in\left(\frac{1}{2}, 1\right) .
$$

Similarly, we have

$$
\left|\left\langle\mathcal{L}_{2,3}^{\Delta}, \phi\right\rangle\right|,\left|\left\langle\mathcal{L}_{2,4}^{\Delta}, \phi\right\rangle\right| \leq C \Delta x^{\alpha-\frac{1}{2}}\|\phi\|_{C_{0}^{\alpha}(\Omega)}, \quad \phi \in C_{0}^{\alpha}(\Omega), \alpha \in\left(\frac{1}{2}, 1\right) .
$$

Sobolev's imbedding theorem gives $W_{0}^{1, p}(\Omega) \subset C_{0}^{\alpha}(\Omega)$ for $\alpha \in\left(0,1-\frac{2}{p}\right)$. Hence

$$
\left|\left\langle\mathcal{L}_{2,2}^{\Delta}, \phi\right\rangle\right|,\left|\left\langle\mathcal{L}_{2,3}^{\Delta}, \phi\right\rangle\right|,\left|\left\langle\mathcal{L}_{2,4}^{\Delta}, \phi\right\rangle\right| \leq C \Delta x^{\alpha-\frac{1}{2}}\|\phi\|_{W_{0}^{1, p}(\Omega)},
$$

for $p>\frac{2}{2-\alpha}, \alpha \in\left(\frac{1}{2}, 1\right)$, that is,

$$
\left\|\mathcal{L}_{2,2}^{\Delta}\right\|_{W^{-1, p}(\Omega)},\left\|\mathcal{L}_{2,3}^{\Delta}\right\|_{W^{-1, p}(\Omega)},\left\|\mathcal{L}_{2,4}^{\Delta}\right\|_{W^{-1, p}(\Omega)} \leq C \Delta x^{\alpha-\frac{1}{2}} \rightarrow 0 \quad \text { as } \Delta \downarrow 0 .
$$

This implies that

$$
\left\{\mathcal{L}_{2,2}^{\Delta}+\mathcal{L}_{2,3}^{\Delta}+\mathcal{L}_{2,4}^{\Delta}\right\}_{\Delta>0} \quad \text { is compact in } \quad W^{-1, q_{3}}(\Omega),
$$

for $q_{3} \in\left(1, \frac{2}{1+\alpha}\right), \alpha \in\left(\frac{1}{2}, 1\right)$.

Summing up, from (4.11), (4.17), and (4.18), it follows that the sequence of distributions $\left\{\mathcal{L}^{\Delta}\right\}_{\Delta>0}$ is compact in $W^{-1, q}(\Omega)$ for $1<q:=\min \left(q_{0}, q_{1}, q_{2}\right)<\frac{2}{1+\alpha}<2$. In addition, since $a \leq u^{\Delta} \leq b,\left\{\mathcal{L}^{\Delta}\right\}_{\Delta>0}$ is bounded in $W^{-1, r}(\Omega)$ for any $r>1$. From Lemma 3.4 we conclude that $\left\{\mathcal{L}^{\Delta}\right\}_{\Delta>0}$ is compact in $W^{-1,2}(\Omega)$. This concludes the proof of the lemma since $\Omega$ was an arbitrary bounded open subset of $\mathbb{R} \times \mathbb{R}_{+}$.

Now we come to our main convergence/existence theorem. 
Theorem 4.5 (Convergence/existence). Suppose (2.1)-(2.6) hold. Let $u^{\Delta}=u^{\Delta}(x, t)$ be the LaxFriedrichs approximate solution generated by (2.7), (2.8), (2.10) and (2.11). Passing if necessary to a subsequence, we have

$$
u^{\Delta} \rightarrow u \quad \text { in } L_{\mathrm{loc}}^{p}\left(\mathbb{R} \times \mathbb{R}_{+}\right) \text {as } \Delta \downarrow 0, \text { for any } p<\infty,
$$

and $u \in L^{\infty}\left(\mathbb{R} \times \mathbb{R}_{+}\right)$is a weak solution $u$ of the Cauchy problem (1.1), i.e., $u$ is a bounded measurable function satisfying $\forall \phi \in \mathcal{D}(\mathbb{R} \times[0, \infty))$

$$
\int_{\mathbb{R}_{+}} \int_{\mathbb{R}}\left(u \phi_{t}+f(k(x, t), u) \phi_{x}\right) d x d t+\int_{\mathbb{R}} u_{0}(x) \phi(x, 0) d x=0 .
$$

Proof. The strong $L_{\text {loc }}^{p}$ - convergence of $u^{\Delta}$ to a function $u \in L^{\infty}\left(\mathbb{R} \times \mathbb{R}_{+}\right)$follows immediately from Lemma 4.4 and Lemma 3.2. It remains to prove that $u$ is a weak solution. Omitting the details, this can be done by taking $S(u)=u, Q(k, u)=f(k, u)$ in the proof of Lemma 4.4.

\section{A KruŽKov type entropy InEQUALITY}

In [14], we proposed a notion of entropy solution for (one-dimensional) degenerate parabolic equations with coefficients that were only spatially dependent. We then showed $L^{1}$ stability and uniqueness of entropy solutions, assuming that a certain crossing condition (see Section 6) is satisfied, and that the solution has traces along the jumps in the discontinuous parameters. The key ingredient of the entropy concept in that paper is a generalization of the classical Kružkov entropy inequality. That generalization includes new terms that account for the contribution at each jump in the spatially discontinuous coefficients. In this section, we introduce a version of that entropy inequality for (1.1), i.e., for the purely hyperbolic problem when the coefficient is allowed to vary temporally as well as spatially.

Before proceeding, we make some additional regularity assumptions about the coefficient $k(x, t)$. We basically assume that $k$ is piecewise Lipschitz continuous in $\mathbb{R} \times[0, \infty)$. More specifically, we assume that there are finitely many Lipschitz continuous curves $\omega_{1}, \omega_{2}, \ldots, \omega_{M}$, whose union we denote

$$
\bigcup_{m=1}^{M} \omega_{m}=\Omega
$$

We assume that each curve $\omega_{m}$ has a Lipschitz continuous parameterization in terms of $t$, i.e.,

$$
x=\xi_{m}(t), \quad t \in[0, \infty), \quad \xi_{m} \in \operatorname{Lip}([0, \infty)), \quad 1 \leq m \leq M .
$$

For such a curve $\omega_{m}$, we denote the speed of the discontinuity by

$$
s_{m}(t)=\frac{d}{d t} \xi_{m}(t)
$$

which is well defined for a.e. $t \in(0, \infty)$. Note that we are not allowing any of the curves $\omega_{m}$ to have any horizontal segments in the $x-t$ plane. For the sake of simplicity, we also assume that none of the curves intersect. The curves $\omega_{1}, \ldots, \omega_{M}$ partition $\mathbb{R} \times \mathbb{R}_{+} \backslash \Omega$ in an obvious way into a finite union of open sets:

$$
\mathbb{R} \times \mathbb{R}_{+} \backslash \Omega=\mathcal{R}_{0} \cup \mathcal{R}_{1} \cup \cdots \cup \mathcal{R}_{M}
$$

with the curve $\omega_{m}$ separating the sets $\mathcal{R}_{m-1}$ and $\mathcal{R}_{m}$. We will assume that

$$
k \in \operatorname{Lip}\left(\overline{\mathcal{R}}_{m}\right), \quad m=1, \ldots, M .
$$

With this assumption, $k$ has well defined limits from the right and left along each of the curves $\omega_{m}, 1 \leq m \leq M$, and we denote these limits by $k\left(\xi_{m}^{ \pm}(t), t\right)$, respectively. By way of simplifying the appearance of the equations, we will sometimes use the notation

$$
k_{m}^{ \pm}(t):=k\left(\xi_{m}^{ \pm}(t), t\right)
$$


With our additional regularity assumptions about the coefficient $k$, we can be more specific about its discretization. In what follows, we will assume that $k$ is discretized via

$$
k^{\Delta}(x, t)=\sum_{n \in \mathbb{Z}_{+}^{0}} \sum_{\substack{j \in \mathbb{Z} \\ j+n=\text { even }}} \chi_{j}^{n}(x, t) k_{j}^{n}, \quad k_{j}^{n}=\lim _{x \downarrow x_{j}} k\left(x_{j}, t_{n}\right) .
$$

This definition is a particular instance of the discretization originally defined by (2.10).

Definition 5.1 (entropy solution). A weak solution $u(x, t)$ of the Cauchy problem (1.1) is called an entropy solution if the following Kružkov-type entropy inequality holds for all $c \in \mathbb{R}$ and all test functions $0 \leq \psi \in \mathcal{D}(\mathbb{R} \times[0, \infty))$ :

$$
\begin{aligned}
\iint_{\mathbb{R}^{\times} \mathbb{R}_{+}} & \left(|u-c| \psi_{t}+\operatorname{sign}(u-c)(f(k, u)-f(k, c)) \psi_{x}\right) d x d t \\
& +\int_{\mathbb{R}}\left|u_{0}-c\right| \psi(x, 0) d x-\iint_{\mathbb{R} \times \mathbb{R}_{+} \backslash \Omega} \operatorname{sign}(u-c) f(k(x, t), c)_{x} \psi d x d t \\
& +\sum_{m=1}^{M} \int_{0}^{\infty}\left|f\left(k_{m}^{+}(t), c\right)-f\left(k_{m}^{-}(t), c\right)\right| \psi\left(\xi_{m}(t), t\right) d t \geq 0 .
\end{aligned}
$$

We now demonstrate via a sequence of lemmas (culminating in Theorem 5.5) that limit solutions constructed via the Lax-Friedrichs scheme are entropy solutions in the sense of Definition 5.1 above. We begin with a discrete entropy inequality established in [14] for the Engquist-Osher scheme. The calculation in that paper is easily adapted to our Lax-Friedrichs algorithm, giving the following lemma, which we state without proof.

Lemma 5.2. For fixed $c \in \mathbf{R}$, let $F(k, u, c):=\operatorname{sign}(u-c)(f(k, u)-f(k, c))$ denote the Kružkov entropy flux associated with the entropy $|u-c|$. The following cell entropy inequality is satisfied by approximate solutions $\left\{U_{j}^{n}\right\}$ generated by the scheme (2.11):

$$
\begin{aligned}
\left|U_{j}^{n+1}-c\right| \leq \frac{1}{2} \mid & U_{j+1}^{n}-c\left|+\frac{1}{2}\right| U_{j-1}^{n}-c \mid \\
-\frac{\lambda}{2}( & \left.F\left(k_{j+1}^{n}, U_{j+1}^{n}, c\right)-F\left(k_{j-1}^{n}, U_{j-1}^{n}, c\right)\right) \\
& \quad-\frac{\lambda}{2} \operatorname{sign}\left(U_{j}^{n+1}-c\right)\left(f\left(k_{j+1}^{n}, c\right)-f\left(k_{j-1}^{n}, c\right)\right) .
\end{aligned}
$$

The next lemma gives the simplified entropy condition that results when the test function has support that does not intersect any of the jump curves $\omega_{1}, \ldots, \omega_{M}$.

Lemma 5.3. Let $u(x, t)$ be a weak solution constructed as the limit of approximations $u^{\Delta}$ generated by the Lax-Friedrichs scheme, as in Theorem 4.5, and let $c \in \mathbb{R}$. Let $0 \leq \psi \in \mathcal{D}(\mathbb{R} \times[0, \infty) \backslash \Omega)$. Then the following entropy inequality is satisified:

$$
\begin{aligned}
& \iint_{\mathbb{R} \times \mathbb{R}_{+}}\left(|u-c| \psi_{t}+\operatorname{sign}(u-c)(f(k, u)-f(k, c)) \psi_{x}\right) d x d t \\
& \quad+\int_{\mathbb{R}}\left|u_{0}-c\right| \psi(x, 0) d x+\iint_{\mathbb{R}^{\prime} \mathbb{R}_{+}} \operatorname{sign}(u-c) f(k(x, t), c)_{x} \psi d x d t \geq 0 .
\end{aligned}
$$

Proof. Let $\psi$ be a test function of the type described in the statement of the lemma, and choose $T>0$ and $X>0$ such that for $\Delta x$ and $\Delta t$ sufficiently small

$$
\operatorname{supp}(\psi) \subseteq[-X+2 \Delta x, X-2 \Delta x] \times[0, T-\Delta t] \subset[-X, X] \times[0, T] .
$$

To simplify the notation, let $V_{j}^{n}=\left|U_{j}^{n}-c\right|, F_{j}^{n}=F\left(k_{j}^{n}, U_{j}^{n}, c\right)$, with $F$ defined in Lemma 5.2, and $\psi_{j}^{n}=\psi\left(x_{j}, t_{n}\right)$. Multiplying (5.4) by $\psi_{j}^{n+1} / \Delta t$, and rearranging, we find that

$$
\begin{aligned}
& \frac{1}{2 \Delta t}\left(V_{j}^{n+1}-V_{j-1}^{n}\right) \psi_{j}^{n+1}+\frac{1}{2 \Delta t}\left(V_{j}^{n+1}-V_{j+1}^{n}\right) \psi_{j}^{n+1}+\frac{1}{2 \Delta x}\left(F_{j+1}^{n}-F_{j-1}^{n}\right) \psi_{j}^{n+1} \\
& \quad+\frac{1}{2 \Delta x} \operatorname{sign}\left(U_{j}^{n+1}-c\right)\left(f\left(k_{j+1}^{n}, c\right)-f\left(k_{j-1}^{n}, c\right)\right) \psi_{j}^{n+1} \leq 0 .
\end{aligned}
$$


We next multiply by $2 \Delta x \Delta t$, and then sum this inequality over $\{(j, n) \mid n \geq 0, j+n+1=$ even $\}$, yielding $\Sigma_{1}+\Sigma_{2}+\Sigma_{3} \leq 0$, where the $\Sigma_{i}$ are defined in (5.7), (5.11), and (5.13) below. We analyze separately the convergence of each of the sums $\Sigma_{1}, \Sigma_{2}, \Sigma_{3}$. First, consider the sum

$$
\Sigma_{1}:=2 \Delta x \Delta t \sum_{n \geq 0} \sum_{\substack{j \in \mathbb{Z} \\ j+n+1=\text { even }}} \frac{1}{2 \Delta x}\left(F_{j+1}^{n}-F_{j-1}^{n}\right) \psi_{j}^{n+1} .
$$

We replace $\psi_{j}^{n+1}$ by $\psi_{j-1}^{n}$ and obtain

$$
\Sigma_{1}=2 \Delta x \Delta t \sum_{n \geq 0} \sum_{\substack{j \in \mathbb{Z} \\ j+n+1=\text { even }}} \frac{1}{2 \Delta x}\left(F_{j+1}^{n}-F_{j-1}^{n}\right) \psi_{j-1}^{n}+E_{1},
$$

where

$$
E_{1}:=2 \Delta x \Delta t \sum_{n \geq 0} \sum_{\substack{j \in \mathbb{Z} \\ j+n+1=\text { even }}} \frac{1}{2 \Delta x}\left(F_{j+1}^{n}-F_{j-1}^{n}\right)\left(\psi_{j}^{n+1}-\psi_{j-1}^{n}\right) .
$$

We claim that $E_{1} \rightarrow 0$ as $\Delta \rightarrow 0$. Clearly,

$$
\left|\psi_{j}^{n+1}-\psi_{j-1}^{n}\right| \leq\left\|\psi_{x}\right\|_{\infty} \Delta x+\left\|\psi_{t}\right\|_{\infty} \Delta t
$$

and so with the notation

$$
\widehat{F}(x, t)=F(k(x, t), u(x, t), c), \quad \widehat{F}^{\Delta}(x, t)=F\left(k^{\Delta}(x, t), u^{\Delta}(x, t), c\right),
$$

we have the following bound for $E_{1}$ :

$$
E_{1} \leq \frac{1}{2}\left(\left\|\psi_{x}\right\|_{\infty}+\lambda\left\|\psi_{t}\right\|_{\infty}\right) \int_{0}^{T} \int_{-X}^{X}\left|\widehat{F}^{\Delta}(x+2 \Delta x, t)-\widehat{F}^{\Delta}(x, t)\right| d x d t .
$$

We use the triangle inequality to estimate this last integral:

$$
\begin{gathered}
\int_{0}^{T} \int_{-X}^{X}\left|\widehat{F}^{\Delta}(x+2 \Delta x, t)-\widehat{F}^{\Delta}(x, t)\right| d x d t \\
\leq \int_{0}^{T} \int_{-X}^{X}\left|\widehat{F}^{\Delta}(x+2 \Delta x, t)-\widehat{F}(x+2 \Delta x, t)\right| d x d t \\
\quad+\int_{0}^{T} \int_{-X}^{X}|\widehat{F}(x+2 \Delta x, t)-\widehat{F}(x, t)| d x d t \\
\quad+\int_{0}^{T} \int_{-X}^{X}\left|\widehat{F}(x, t)-\widehat{F}^{\Delta}(x, t)\right| d x d t .
\end{gathered}
$$

Since $F$ is Lipshitz-continuous, convergence in $L_{\text {loc }}^{p}\left(\mathbb{R} \times \mathbb{R}_{+}\right)$of $k^{\Delta}$ and $u^{\Delta}$ implies that $\widehat{F}^{\Delta} \rightarrow \widehat{F}$ in $L_{\mathrm{loc}}^{p}\left(\mathbb{R} \times \mathbb{R}_{+}\right)$. Thus, the first and third integrals on the right side of (5.9) converge to zero as $\Delta \rightarrow 0$. By a standard fact from real analysis, the second integral also converges to zero, and so we see that also $E_{1} \rightarrow 0$. Using summation by parts, along with the bounded convergence theorem, it is now evident that

$$
\Sigma_{1} \rightarrow-\iint_{\mathbb{R} \times \mathbb{R}_{+}} F(u, k, c) \psi_{x} d x d t
$$


Next, consider the sum

$$
\begin{aligned}
& \Sigma_{2}:=2 \Delta x \Delta t \sum_{n \geq 0} \sum_{\substack{j \in \mathbb{Z} \\
j+n+1=\text { even }}}\left(\frac{1}{2 \Delta t}\left(V_{j}^{n+1}-V_{j-1}^{n}\right) \psi_{j}^{n+1}+\frac{1}{2 \Delta t}\left(V_{j}^{n+1}-V_{j+1}^{n}\right) \psi_{j}^{n+1}\right) \\
&=\Delta x \Delta t \sum_{n \geq 0} \sum_{\substack{j \in \mathbb{Z} \\
j+n+1=\text { even }}} \frac{-1}{\Delta t}\left(V_{j-1}^{n}\left(\psi_{j}^{n+1}-\psi_{j-1}^{n}\right)+V_{j+1}^{n}\left(\psi_{j}^{n+1}-\psi_{j+1}^{n}\right)\right) \\
&+2 \Delta x \sum_{\substack{j \in \mathbb{Z} \\
j+1=\operatorname{even}}}\left(\frac{V_{j-1}^{0}+V_{j+1}^{0}}{2}\right) \psi_{j}^{1} \\
&=-2 \Delta x \Delta t \sum_{n \geq 0} \sum_{j \in \mathbb{Z}} V_{j+1}^{n}\left(\frac{\frac{1}{2} \psi_{j}^{n+1}+\frac{1}{2} \psi_{j+2}^{n+1}-\psi_{j+1}^{n}}{\Delta t}\right) \\
&+2 \Delta x \sum_{j \in \mathbb{Z}}\left(\frac{V_{j-1}^{0}+V_{j+1}^{0}}{2}\right) \psi_{j}^{1} .
\end{aligned}
$$

The quantity in parentheses in the first sum on the last line is equal to $\psi_{t}\left(x_{j+1}, t^{n}\right)+\mathcal{O}\left(\Delta^{2}\right)$, and so an application of the bounded convergence theorem gives

$$
\Sigma_{2} \rightarrow-\iint_{\mathbb{R} \times \mathbb{R}_{+}}|u-c| \psi_{t} d x d t-\int_{\mathbb{R}}\left|u_{0}-c\right| \psi(x, 0) d x .
$$

Finally, we address the sum

$$
\Sigma_{3}:=2 \Delta x \Delta t \sum_{n \geq 0} \sum_{\substack{j \in \mathbb{Z} \\ j+n+1=\text { even }}} \frac{1}{2 \Delta x} \operatorname{sign}\left(U_{j}^{n+1}-c\right)\left(f\left(k_{j+1}^{n}, c\right)-f\left(k_{j-1}^{n}, c\right)\right) \psi_{j}^{n+1} .
$$

By the fact that $k$ is Lipschitz continuous within the support of $\psi$, we find (by applying Lemma 4.3 of [14]) that there is a set $\Theta$ which is at most countable such that for $c \in \mathbb{R} \backslash \Theta$,

$$
\Sigma_{3} \rightarrow \iint_{\mathbb{R}_{\mathbb{R}_{+}}} \operatorname{sign}(u-c) f(k(x, t), c)_{x} \psi d x d t
$$

Combining (5.10), (5.12), and (5.14), we conclude that (5.5) holds for $c \in \mathbb{R} \backslash \Theta$. To complete the proof, we must show that (5.5) actually holds for all $c \in \mathbb{R}$. For this, we can proceed as in the proof of Lemma 4.4 of [14], to which we refer the interested reader.

Lemma 5.4. Let $u(x, t)$ be a weak solution constructed as the limit of approximations $u^{\Delta}$ generated by the Lax-Friedrichs scheme, as in Theorem 4.5. Let $0 \leq \psi \in \mathcal{D}(\mathbb{R} \times[0, \infty))$. Then the following entropy inequality is satisfied for all $c \in \mathbb{R}$ :

$$
\begin{aligned}
\iint_{\mathbb{R}^{\prime} \times \mathbb{R}_{+}} & \left(|u-c| \phi_{t}+\operatorname{sign}(u-c)(f(k, u)-f(k, c)) \psi_{x}\right) d x d t \\
+ & \int_{\mathbb{R}}\left|u_{0}-c\right| \psi(x, 0) d x+\iint_{\mathbb{R} \times \mathbb{R}_{+} \backslash \Omega}\left|f(k(x, t), c)_{x}\right| \psi d x d t \\
& \quad+\sum_{n=1}^{M} \int_{0}^{\infty}\left|f\left(k_{m}^{+}(t), c\right)-f\left(k_{m}^{-}(t), c\right)\right| \psi\left(\xi_{m}(t), t\right) d t \geq 0 .
\end{aligned}
$$

Proof. For now, assume that the support of $\psi$ intersects at most one of the curves, say $\omega_{m}$, but none of the other jump curves. 
Our starting point is the following cell entropy inequality, which is a simple consequence of the cell entropy inequality (5.4).

$$
\begin{aligned}
&\left|U_{j}^{n+1}-c\right| \leq \frac{1}{2} \mid U_{j+1}^{n}-c\left|+\frac{1}{2}\right| U_{j-1}^{n}-c \mid \\
&-\frac{\lambda}{2}\left(F\left(k_{j+1}^{n}, U_{j+1}^{n}, c\right)-F\left(k_{j-1}^{n}, U_{j-1}^{n}, c\right)\right) \\
& \quad+\frac{\lambda}{2}\left|f\left(k_{j+1}^{n}, c\right)-f\left(k_{j-1}^{n}, c\right)\right| .
\end{aligned}
$$

Proceeding as in the proof of Lemma 5.3, we multiply the entropy inequality (5.16) by $\psi_{j}^{n+1} / \Delta t$, rearrange, then multiply by $2 \Delta x \Delta t$, and sum over the set $\{(j, n) \mid n \geq 0, j+n+1=$ even $\}$, resulting in $\Sigma_{1}+\Sigma_{2}+\widetilde{\Sigma}_{3} \leq 0$, where $\Sigma_{1}$ and $\Sigma_{2}$ are defined by (5.7) and (5.11), and

$$
\widetilde{\Sigma}_{3}:=2 \Delta x \Delta t \sum_{n \geq 0} \sum_{\substack{j \in \mathbb{Z} \\ j+n+1=\text { even }}}\left|\frac{f\left(k_{j+1}^{n}, c\right)-f\left(k_{j-1}^{n}, c\right)}{2 \Delta x}\right| \psi_{j}^{n+1} .
$$

We can repeat the arguments used in Lemma 5.3 to show that

$$
\Sigma_{1} \rightarrow-\iint_{\mathbb{R} \times \mathbb{R}_{+}} \operatorname{sign}(u-c)(f(k, u)-f(k, c)) \psi_{x} d x d t-\int_{\mathbb{R}}\left|u_{0}-c\right| \psi(x, 0) d x,
$$

and

$$
\Sigma_{2} \rightarrow-\iint_{\mathbb{R}_{\mathbb{R}}}|u-c| \psi_{t} d x d t
$$

We now address the sum $\widetilde{\Sigma}_{3}$. Due to our regularity assumptions and method of discretizing $k$, there is a unique index $j_{0}=j_{0}(n)$ such that

$$
x_{j_{0}-1}<\xi_{m} \leq x_{j_{0}+1}
$$

and

$$
\begin{aligned}
& \left|k_{m}^{-}\left(t^{n}\right)-k_{j_{0}-1}^{n}\right| \leq 2 \Delta x\left\|k_{x}\right\|_{L^{\infty}\left(\mathbb{R} \times \mathbb{R}_{+} \backslash \Omega\right)}, \\
& \left|k_{m}^{+}\left(t^{n}\right)-k_{j_{0}+1}^{n}\right| \leq 2 \Delta x\left\|k_{x}\right\|_{L^{\infty}\left(\mathbb{R} \times \mathbb{R}_{+} \backslash \Omega\right)} .
\end{aligned}
$$

Next, we write $\widetilde{\Sigma}_{3}$ in the form

$$
\begin{aligned}
\widetilde{\Sigma}_{3}:=2 \Delta & x \Delta t \sum_{n \geq 0}\left|\frac{f\left(k_{j_{0}+1}^{n}, c\right)-f\left(k_{j_{0}-1}^{n}, c\right)}{2 \Delta x}\right| \psi_{j_{0}}^{n+1} \\
& +2 \Delta x \Delta t \sum_{n \geq 0} \sum_{\substack{j<j_{0} \\
j+n+1=\text { even }}}\left|\frac{f\left(k_{j+1}^{n}, c\right)-f\left(k_{j-1}^{n}, c\right)}{2 \Delta x}\right| \psi_{j}^{n+1} \\
& +2 \Delta x \Delta t \sum_{n \geq 0} \sum_{\substack{j>j_{0} \\
j+n+1=\text { even }}}\left|\frac{f\left(k_{j+1}^{n}, c\right)-f\left(k_{j-1}^{n}, c\right)}{2 \Delta x}\right| \psi_{j}^{n+1} \\
= & : \widetilde{\Sigma}_{31}+\widetilde{\Sigma}_{32}+\widetilde{\Sigma}_{33} .
\end{aligned}
$$

By applying the bounded convergence theorem we obtain

$$
\widetilde{\Sigma}_{32}+\widetilde{\Sigma}_{33} \rightarrow \iint_{\mathbb{R} \times \mathbb{R}_{+} \backslash \omega_{m}}\left|f(k(x, t), c)_{x}\right| \psi d x d t .
$$

Recalling (5.20), we can write $\widetilde{\Sigma}_{31}$ in the form

$$
\widetilde{\Sigma}_{31}=\Delta t \sum_{n \geq 0}\left|f\left(k_{m}^{+}\left(t^{n}\right), c\right)-f\left(k_{m}^{-}\left(t^{n}\right), c\right)\right| \psi_{j_{0}}^{n+1}+E_{2}(\Delta x),
$$

where

$$
\left|E_{2}(\Delta x)\right| \leq 4 T\left\|f_{k}\right\|_{\infty}\left\|k_{x}\right\|_{L^{\infty}\left(\mathbb{R} \times \mathbb{R}_{+} \backslash \Omega\right)}\|\psi\|_{\infty} \Delta x
$$


From this it is clear that as $\Delta \rightarrow 0$,

$$
\widetilde{\Sigma}_{31} \rightarrow \int_{0}^{\infty}\left|f\left(k_{m}^{+}(t), c\right)-f\left(k_{m}^{-}(t), c\right)\right| \psi\left(\xi_{m}(t), t\right) d t
$$

By combining (5.18), (5.19), (5.22), and (5.23), we conclude that the desired entropy inequality (5.15) holds in the case where the support of $\psi$ intersects only one jump curve.

For the general case, we can decompose the test function $0 \leq \psi \in \mathcal{D}(\mathbb{R} \times[0, \infty))$ according to

$$
\psi=\psi_{1}+\cdots+\psi_{M}, \quad 0 \leq \psi_{m} \in \mathcal{D}(\mathbb{R} \times[0, \infty)), \quad m=1, \ldots, M,
$$

where $\operatorname{supp}\left(\psi_{m}\right)$ possibly intersects $\omega_{m}$, but none of the other jump curves. By the preceding argument, the entropy inequality (5.15) holds for each $\psi_{m}$ separately. By adding each of the resulting entropy inequalities, we then conclude that (5.15) holds for $\psi$.

Theorem 5.5. Suppose (2.1)-(2.6) hold, and that $k$ satisfies the additional regularity conditions described at the beginning of this section. Let $u^{\Delta}=u^{\Delta}(x, t)$ be the Lax-Friedrichs approximate solution generated by (2.7), (2.8), and (2.11), using (5.2) to discretize $k$. Let $u: \mathbb{R} \times \mathbb{R}_{+} \rightarrow \mathbb{R}$ be a weak solution constructed as the limit of a subsequence of the approximations $u^{\Delta}$, as in Theorem 4.5. Then $u$ is an entropy solution.

Proof. Let $0 \leq \psi \in \mathcal{D}(\mathbb{R} \times[0, \infty))$. For $\varepsilon>0$, define the tube $\omega_{m}^{\varepsilon}$ of width $2 \varepsilon$ containing the curve $\omega_{m}$ :

$$
\omega_{m}^{\varepsilon}:=\left\{(x, t) \in \mathbb{R} \times[0, \infty) \mid x \in\left(\xi_{m}(t)-\varepsilon, \xi_{m}(t)+\varepsilon\right), t \in[0, \infty)\right\} .
$$

For each sufficiently small $\varepsilon>0$ we can write the test function $\psi$ as a sum of two test functions, one having support away from the set $\Omega$, and the other with support in the vicinity of $\Omega$. Concretely, there are test functions $\rho^{\varepsilon}, \sigma^{\varepsilon} \in \mathcal{D}(\mathbb{R} \times[0, \infty))$ such that

$$
\psi(x, t)=\rho^{\varepsilon}(x, t)+\sigma^{\varepsilon}(x, t), \quad 0 \leq \rho^{\varepsilon}(x, t) \leq \psi(x, t), \quad 0 \leq \sigma^{\varepsilon}(x, t) \leq \psi(x, t)
$$

where $\rho^{\varepsilon}$ has support located around the jumps in $k$ :

$$
\begin{gathered}
\operatorname{supp}\left(\rho^{\varepsilon}\right) \subseteq \omega_{1}^{\varepsilon} \cup \cdots \cup \omega_{M}^{\varepsilon}, \\
\rho^{\varepsilon}\left(\xi_{m}(t), t\right)=\psi\left(\xi_{m}(t), t\right), \quad m=1, \ldots, M, \quad \varepsilon>0,
\end{gathered}
$$

and $\sigma^{\varepsilon}$ vanishes around the jumps in $k$, i.e.,

$$
\operatorname{supp}\left(\sigma^{\varepsilon}\right) \subseteq(\mathbb{R} \times[0, \infty) \backslash \Omega) .
$$

We can accomplish this decomposition in such a way that

$$
\sigma^{\varepsilon} \rightarrow \psi \text { in } L^{1}\left(\mathbb{R} \times \mathbb{R}_{+}\right), \quad \rho^{\varepsilon} \rightarrow 0 \text { in } L^{1}\left(\mathbb{R} \times \mathbb{R}_{+}\right) .
$$

By applying Lemma 5.3 with the test function $\sigma^{\varepsilon}$, we obtain

$$
\begin{aligned}
& \iint_{\mathbb{R}^{\prime} \times \mathbb{R}_{+}}\left(|u-c| \sigma_{t}^{\varepsilon}+\operatorname{sign}(u-c)(f(k, u)-f(k, c)) \sigma_{x}^{\varepsilon}\right) d x d t \\
& \quad+\int_{\mathbb{R}^{\prime}}\left|u_{0}(x)-c\right| \sigma^{\varepsilon}(x, 0) d x-\iint_{\mathbb{R}^{\prime} \times \mathbb{R}_{+} \backslash \Omega} \operatorname{sign}(u-c) f(k(x, t), c)_{x} \sigma^{\varepsilon} d x d t \geq 0 .
\end{aligned}
$$

Similarly, an application of Lemma 5.4 with the test function $\rho^{\varepsilon}$ yields

$$
\begin{aligned}
& \iint_{\mathbb{R}^{\times} \mathbb{R}_{+}}\left(|u-c| \rho_{t}^{\varepsilon}+\operatorname{sign}(u-c)(f(k, u)-f(k, c)) \rho_{x}^{\varepsilon}\right) d x d t \\
& \quad+\int_{\mathbb{R}^{\prime}}\left|u_{0}(x)-c\right| \rho^{\varepsilon}(x, 0) d x+\iint_{\mathbb{R} \times \mathbb{R}_{+} \backslash \Omega}\left|f(k(x, t), c)_{x}\right| \rho^{\varepsilon} d x d t \\
& \quad+\sum_{m=1}^{M} \int_{0}^{\infty}\left|f\left(k\left(\xi_{m}^{+}(t), t\right), c\right)-f\left(k\left(\xi_{m}^{-}(t), t\right), c\right)\right| \rho^{\varepsilon}\left(\xi_{m}(t), t\right) d t \geq 0 .
\end{aligned}
$$


We now add the two entropy inequalities (5.25) and (5.26), using $\rho^{\varepsilon}+\sigma^{\varepsilon}=\psi$, along with $\rho^{\varepsilon}\left(\xi_{m}(t), t\right)=\psi\left(\xi_{m}(t), t\right)$ to get

$$
\begin{aligned}
& \iint_{\mathbb{R} \times \mathbb{R}_{+}}\left(|u-c| \psi_{t}+\operatorname{sign}(u-c)(f(k, u)-f(k, c)) \psi_{x}\right) d t d x \\
& +\int_{\mathbb{R}^{\prime}}\left|u_{0}(x)-c\right| \psi(x, 0) d x+\iint_{\mathbb{R}^{\prime} \times \mathbb{R}_{+} \backslash \Omega}\left|f(k, c)_{x}\right| \rho^{\varepsilon} d t d x \\
& \quad-\iint_{\mathbb{R}^{\prime} \mathbb{R}_{+}} \operatorname{sign}(u-c) f(k, c)_{x} \sigma^{\varepsilon} d t d x \\
& \quad+\sum_{m=1}^{M} \int_{0}^{\infty}\left|f\left(k_{m}^{+}(t), c\right)-f\left(k_{m}^{-}(t), c\right)\right| \psi\left(\xi_{m}(t), t\right) d t \geq 0 .
\end{aligned}
$$

Thanks to (5.24), we complete the proof by sending $\varepsilon \downarrow 0$ in (5.27).

\section{A uniqueness Result}

As we mentioned previously, in [14] we proved $L^{1}$ stability and uniqueness of entropy solutions, assuming that the coefficient was independent of time. In this section we indicate how those results can be extended to the situation considered here, i.e., $k(x, t)$ depends on both space and time. Throughout this section we continue to assume that $k$ satisfies the additional regularity conditions described at the beginning of Section 5 .

As in [14], we impose a so-called crossing condition on the flux at each discontinuity in $k$. By way of describing that condition, fix a jump in $k(x, t)$ located at a point $\left(\xi_{m}(t), t\right)$ on one of the curves $\omega_{m}, 1 \leq m \leq M$. Let us temporarily suppress the dependence on time, writing $k_{m}^{\mp}:=k_{m}^{\mp}(t)$, and observe that the graphs of $u \mapsto f\left(k_{m}^{-}, u\right)$ and $u \mapsto f\left(k_{m}^{+}, u\right)$ can cross. For such a crossing, we make the following assumption:

Assumption 6.1 (crossing condition). For any jump in $k$ with associated left and right limits $\left(k_{m}^{-}, k_{m}^{+}\right)$, we require that for any states $u$ and $v$, the following crossing condition must hold:

$$
f\left(k_{m}^{+}, u\right)-f\left(k_{m}^{-}, u\right)<0<f\left(k_{m}^{+}, v\right)-f\left(k_{m}^{-}, v\right) \Longrightarrow u<v .
$$

Geometrically, the crossing condition requires that either the graphs of $f\left(k_{m}^{-}, \cdot\right)$ and $f\left(k_{m}^{+}, \cdot\right)$ do not cross, or if they do, the graph of $f\left(k_{m}^{-}, \cdot\right)$ lies above the graph of $f\left(k_{m}^{+}, \cdot\right)$ to the left of any crossing point. As mentioned in [14], our crossing condition rules out some physically important examples. Nevertheless, there are also important cases where the crossing condition is satisfied. One example is the clarifier-thickener model [3], which includes a nontrivial flux crossing that satisfies the crossing condition. Of course, in the multiplicative case $f(k, u)=k f(u)$ there is no flux crossing, and Assumption 6.1 is trivially satisfied.

Remark 6.1. We impose the crossing condition only because the entropy inequality (5.3) is not sufficient to guarantee uniqueness when the crossing condition is violated. We do not intend to convey that only problems satisfying the crossing condition are well posed. In a forthcoming paper [15] we will augment the entropy condition in such a way that uniqueness is assured whether or not the crossing condition is satisfied.

One more technical issue is the existence of traces along the discontinuity curves $\omega_{m}, m=$ $1, \ldots, M$. We make the following assumption.

Assumption 6.2. Let $u$ be an entropy solution of (1.1). For $m=1, \ldots, M$, suppose $u$ admits strong right and left traces along each curve $\omega_{m}$. We denote these traces by $u_{m}^{ \pm}(t):=u\left(\xi_{m}^{ \pm}(t), t\right)$, respectively, and suppose $u_{m}^{ \pm}(\cdot) \in L^{\infty}\left(\omega_{m}\right)$. Hence, for any $T>0$,

$$
\underset{\varepsilon \downarrow 0}{\operatorname{ess} \lim _{0}} \int_{0}^{T}\left|u\left(\xi_{m}(t) \pm \varepsilon, t\right)-u_{m}^{ \pm}(t)\right| d t=0, \quad m=1, \ldots, M .
$$

In [14], we showed in some important examples that with the additional assumption that $\partial_{t} u$ is a Radon measure, it is possible to demonstrate the existence of traces directly from the fact that 
$u$ is an entropy solution. Another instance where we automatically have the existence of strong traces is when $k(x, t)$ is constant on each region $\mathcal{R}_{m}, m=1, \ldots, M$. This is due to the genuinely nonlinearity assumption (2.4), which induces a regularizing effect at the boundary of each $\mathcal{R}_{m}$. This is a consequence of a general result by Vasseur [36].

Lemma 6.2. For $m=1, \ldots, M$, suppose $\mathcal{R}_{m}$ is an open set with a regular Lipschitz boundary. Let $u$ be an entropy solution to the Cauchy problem (1.1). Suppose

$$
k(x, t)=k_{m} \in \mathbb{R}, \quad \forall(x, t) \in \mathcal{R}_{m}, \quad m=1, \ldots, M .
$$

Then $u$ admits strong (right and left) traces $u_{m}^{ \pm}(t)$ along each curve $\omega_{m}, m=1, \ldots, M$.

Moreover, $u$ admits a strong trace at $t=0+$, so that the initial condition $\left.u\right|_{t=0}=u_{0}$ is satisfied in the strong $L_{\mathrm{loc}}^{1}$ sense.

Proof. The proof is simply to observe that we can apply [36, Theorem 1], since Definition 5.1 implies that $u$ satisfies

$$
\left\{\begin{array}{l}
u_{t}+f\left(k_{m}, u\right)_{t}=0, \quad \eta(u)_{t}+q\left(k_{m}, u\right)_{x} \leq 0, \quad \text { in } \mathcal{R}_{m}, \\
\forall \eta \in C^{2}(\mathbb{R}), \eta^{\prime \prime} \geq 0, q_{u}\left(k_{m}, u\right)=\eta^{\prime}(u) f_{u}\left(k_{m}, u\right)
\end{array}\right.
$$

and $\mathcal{R}_{m}$ is an open set with a regular Lipschitz boundary, $m=1, \ldots, M$.

Lemma 6.2 can probably be extended to the case where $k(x, t)$ varies smoothly on each $\mathrm{R}_{m}$ by replacing the kinetic approach used in [36] by the compensated compactness method used herein, but this is outside the scope of the present paper. Here we simply assume the existence of traces in the general case (Assumption 6.2).

Turning now to the goal of establishing uniqueness, we have the following lemma, which provides the Rankine-Hugoniot jump condition and the entropy jump condition along the curves $\omega_{m}$, for $1 \leq m \leq M$.

Lemma 6.3. Let $u$ be an entropy solution. Fix one of the discontinuity curves $\omega_{m}, m=1, \ldots, M$. The following Rankine-Hugoniot condition holds for almost all $t \in \mathbb{R}_{+}$:

$$
f\left(k_{m}^{+}(t), u_{m}^{+}(t)\right)-f\left(k_{m}^{-}(t), u_{m}^{-}(t)\right)=s_{m}(t)\left[u_{m}^{+}(t)-u_{m}^{-}(t)\right],
$$

and the following entropy jump condition holds for almost all $t \in \mathbb{R}_{+}$and for every $c \in \mathbb{R}$ :

$$
\begin{aligned}
& F\left(k_{m}^{+}(t), u_{m}^{+}(t), c\right)-F\left(k_{m}^{-}(t), u_{m}^{-}(t), c\right)-s_{m}(t)\left[\left|u_{m}^{+}(t)-c\right|-\left|u_{m}^{-}(t)-c\right|\right] \\
& \quad \leq\left|f\left(k_{m}^{+}(t), c\right)-f\left(k_{m}^{-}(t), c\right)\right| .
\end{aligned}
$$

Proof. We begin with the proof of (6.3). First, let us define the following compactly supported Lipschitz function $(\varepsilon>0)$

$$
\theta_{\varepsilon}(x)= \begin{cases}\frac{1}{\varepsilon}(\varepsilon+x), & \text { if } x \in[-\varepsilon, 0], \\ \frac{1}{\varepsilon}(\varepsilon-x), & \text { if } x \in[0, \varepsilon], \\ 0, & \text { if }|x| \geq \varepsilon .\end{cases}
$$

Then, since $u(x, t), k(x, t) \in L^{\infty}\left(\mathbb{R} \times \mathbb{R}_{+}\right)$, a density argument reveals that

$$
\phi(x, t)=\theta_{\varepsilon}\left(x-\xi_{m}(t)\right) \varphi(t), \quad \varphi \in \mathcal{D}(0, T),
$$

can be used as an admissible test function in the weak formulation 4.19. The result is

$$
\begin{aligned}
\int_{0}^{\infty} & \int_{\mathbb{R}} u\left(\theta_{\varepsilon}\left(x-\xi_{m}(t)\right) \varphi^{\prime}(t)-s_{m}(t) \theta_{\varepsilon}^{\prime}\left(x-\xi_{m}(t)\right) \varphi(t)\right) d t d x \\
& +\frac{1}{\varepsilon} \int_{0}^{\infty} \int_{\xi_{m}(t)-\varepsilon}^{\xi_{m}(t)} f(k(x, t), u) \varphi(t) d x d t \\
& -\frac{1}{\varepsilon} \int_{0}^{\infty} \int_{\xi_{m}(t)}^{\xi_{m}(t)+\varepsilon} f(k(x, t), u) \varphi(t) d x d t=0 .
\end{aligned}
$$

Here we have used the fact that for a.e. $t \in \mathbb{R}_{+}$

$$
\partial_{t} \phi(x, t)=\theta_{\varepsilon}\left(x-\xi_{m}(t)\right) \varphi^{\prime}(t)-s_{m}(t) \theta_{\varepsilon}^{\prime}\left(x-\xi_{m}(t)\right) \varphi(t) .
$$


As $\varepsilon \downarrow 0$ the integrals on the second and third lines of (6.6) converge according to

$$
\begin{aligned}
\frac{1}{\varepsilon} \int_{0}^{\infty} \int_{\xi_{m}(t)-\varepsilon}^{\xi_{m}(t)} f(k(x, t), u) \varphi(t) d x d t & \rightarrow \int_{0}^{\infty} f\left(k_{m}^{-}(t), u_{m}^{-}(t)\right) \varphi(t) d t, \\
\frac{1}{\varepsilon} \int_{0}^{\infty} \int_{\xi_{m}(t)}^{\xi_{m}(t)+\varepsilon} f(k(x, t), u) \varphi(t) d x d t & \rightarrow \int_{0}^{\infty} f\left(k_{m}^{+}(t), u_{m}^{+}(t)\right) \varphi(t) d t .
\end{aligned}
$$

For the integral on the first line of (6.6),

$$
\begin{aligned}
& \int_{0}^{\infty} \int_{\mathbb{R}} u(x, t)\left(\theta_{\varepsilon}\left(x-\xi_{m}(t)\right) \varphi^{\prime}(t)-s_{m}(t) \theta_{\varepsilon}^{\prime}\left(x-\xi_{m}(t)\right) \varphi(t)\right) d t d x \\
& =\int_{0}^{\infty} \int_{\mathbb{R}} u(x, t) \theta_{\varepsilon}\left(x-\xi_{m}(t)\right) \varphi^{\prime}(t) d t d x \\
& \quad-\int_{0}^{\infty} \int_{\xi_{m}(t)-\varepsilon}^{\xi_{m}(t)} s_{m}(t) u(x, t) \varphi(t) d x d t+\int_{0}^{\infty} \int_{\xi_{m}(t)}^{\xi_{m}(t)+\varepsilon} s_{m}(t) u(x, t) \varphi(t) d x d t .
\end{aligned}
$$

When $\varepsilon \downarrow 0$, the first integral on the right side of (6.8) converges to zero, while the remaining integrals converge to

$$
\int_{0}^{\infty}\left(-s_{m}(t) u_{m}^{+}(t)+s_{m}(t) u_{m}^{-}(t)\right) \varphi(t) d t .
$$

By combining these limits, it is evident that when $\varepsilon \downarrow 0$ in (6.6), the result is

$$
\int_{0}^{\infty}\left(f\left(k_{m}^{+}(t), u_{m}^{+}(t)\right)-f\left(k_{m}^{-}(t), u_{m}^{-}(t)\right)-s_{m}(t) u_{m}^{+}(t)+s_{m}(t) u_{m}^{-}(t)\right) \varphi(t) d t=0 .
$$

Since $\varphi$ is an arbitrary test function on $(0, \infty)$, the integrand must vanish for a.e. $t \in(0, \infty)$, and the proof of $(6.3)$ is complete.

To prove (6.4), we use the test function $\phi$ in the entropy inequality (5.3). The result is

$$
\begin{aligned}
\int_{0}^{\infty} & \int_{\mathbb{R}}|u-c|\left(\theta_{\varepsilon}\left(x-\xi_{m}(t)\right) \varphi^{\prime}(t)-s_{m}(t) \theta_{\varepsilon}^{\prime}\left(x-\xi_{m}(t)\right) \varphi(t)\right) d t d x \\
& +\frac{1}{\varepsilon} \int_{0}^{\infty} \int_{\xi_{m}(t)-\varepsilon}^{\xi_{m}(t)} \operatorname{sign}(u-c)(f(k(x, t), u)-f(k(x, t), c)) \varphi(t) d x d t \\
& -\frac{1}{\varepsilon} \int_{0}^{\infty} \int_{\xi_{m}(t)}^{\xi_{m}(t)+\varepsilon} \operatorname{sign}(u-c)(f(k(x, t), u)-f(k(x, t), c)) \varphi(t) d x d t \\
& -\iint_{\mathbb{R} \times \mathbb{R}_{+} \backslash \Omega} \operatorname{sign}(u-c) f(k(x, t), c)_{x} \phi d x d t \\
& +\int_{0}^{\infty}\left|f\left(k\left(\xi_{m}^{+}, t\right), c\right)-f\left(k\left(\xi_{m}^{-}, t\right), c\right)\right| \varphi(t) d t \geq 0 .
\end{aligned}
$$

Since $\theta^{\varepsilon} \rightarrow 0$ in $L^{1}(\mathbb{R})$ as $\varepsilon \downarrow 0$, we have

$$
\iint_{\mathbb{R} \times \mathbb{R}_{+} \backslash \Omega} \operatorname{sign}(u-c) f(k(x, t), c)_{x} \phi d x d t \rightarrow 0 .
$$

With this in mind, the proof of (6.4) can be completed in a manner similar to the proof of (6.3), and so the details are omitted.

By combining the jump conditions (6.3) and (6.4), we get geometric entropy conditions, which we state in the next lemma.

Lemma 6.4. Let $u$ be an entropy solution. Fix one of the curves of discontinuity, say $\omega_{m}$, $1 \leq m \leq M$. Fix a time $t \in \mathbb{R}_{+}$where $s_{m}(t)$ and the right and left traces $u_{m}^{ \pm}(t)$ exist. Suppressing the dependence on time, let

$$
u_{m}^{\mp}=u_{m}^{\mp}(t), \quad k_{m}^{\mp}=k_{m}^{\mp}(t), \quad s_{m}(t)=s_{m} .
$$

The appropriate inequality in Table 1 holds for all c lying between $u_{m}^{-}$and $u_{m}^{+}$. 


\begin{tabular}{c|cc} 
& $f\left(k_{m}^{-}, c\right) \leq f\left(k_{m}^{+}, c\right)$ & $f\left(k_{m}^{-}, c\right) \geq f\left(k_{m}^{+}, c\right)$ \\
\hline$u_{m}^{-} \leq c \leq u_{m}^{+}$ & $f\left(k_{m}^{+}, u_{m}^{+}\right)+s_{m}\left(c-u_{m}^{+}\right) \leq f\left(k_{m}^{+}, c\right)$ & $f\left(k_{m}^{-}, u_{m}^{-}\right)+s_{m}\left(c-u_{m}^{-}\right) \leq f\left(k_{m}^{-}, c\right)$ \\
$u_{m}^{+} \leq c \leq u_{m}^{-}$ & $f\left(k_{m}^{-}, u_{m}^{-}\right)+s_{m}\left(c-u_{m}^{-}\right) \geq f\left(k_{m}^{-}, c\right)$ & $f\left(k_{m}^{+}, u_{m}^{+}\right)+s_{m}\left(c-u_{m}^{+}\right) \geq f\left(k_{m}^{+}, c\right)$
\end{tabular}

TABlE 1. Entropy jump conditions.

Proof. Let $c$ be a constant lying between $u_{m}^{-}$and $u_{m}^{+}$. With our simplified notation, the RankineHugoniot condition and entropy jump conditions boil down to

$$
f\left(k_{m}^{+}, u_{m}^{+}\right)-f\left(k_{m}^{-}, u_{m}^{-}\right)=s_{m}\left(u_{m}^{+}-u_{m}^{-}\right),
$$

and

$$
F\left(k_{m}^{+}, u_{m}^{+}, c\right)-F\left(k_{m}^{-}, u_{m}^{-}, c\right)-s_{m}\left(\left|u_{m}^{+}-c\right|-\left|u_{m}^{-}-c\right|\right) \leq\left|f\left(k_{m}^{+}, c\right)-f\left(k_{m}^{-}, c\right)\right| .
$$

Now consider the case in the upper left entry of Table 1, i.e., $u_{m}^{-} \leq c \leq u_{m}^{+}, f\left(k_{m}^{-}, c\right) \leq f\left(k_{m}^{+}, c\right)$. Assume for now that $c \in\left(u_{m}^{-}, u_{m}^{+}\right)$. In this case, inequality (6.12) becomes

$$
\begin{aligned}
& f\left(k_{m}^{+}, u_{m}^{+}\right)-f\left(k_{m}^{+}, c\right)+f\left(k_{m}^{-}, u_{m}^{-}\right)-f\left(k_{m}^{-}, c\right) \\
& \quad \leq s_{m}\left(u_{m}^{+}-c+u_{m}^{-}-c\right)+f\left(k_{m}^{+}, c\right)-f\left(k_{m}^{-}, c\right) .
\end{aligned}
$$

Cancelling $f\left(k_{m}^{-}, c\right)$ from both sides, then applying the Rankine-Hugoniot condition (6.11), and finally dividing by two, gives the upper left entry in the table,

$$
f\left(k_{m}^{+}, u_{m}^{+}\right)+s_{m}\left(c-u_{m}^{+}\right) \leq f\left(k_{m}^{+}, c\right) .
$$

Now assume that $c$ coincides with one of the endpoints, say $c=u_{m}^{+}$, the case where $c=u_{m}^{-}$ being similar. Then inequality (6.12) becomes

$$
f\left(k_{m}^{-}, u_{m}^{-}\right)-f\left(k_{m}^{-}, c\right) \leq s_{m}\left(u_{m}^{-}-c\right)+f\left(k_{m}^{+}, c\right)-f\left(k_{m}^{-}, c\right) .
$$

Cancelling $f\left(k_{m}^{-}, c\right)$ from both sides, and using the Rankine-Hugoniot condition (6.11) again gives

$$
f\left(k_{m}^{+}, u_{m}^{+}\right) \leq f\left(k_{m}^{+}, c\right) .
$$

With the assumption that $c=u_{m}^{+}$we have $s_{m}\left(c-u_{m}^{+}\right)=0$, and so (6.13) holds in this case also.

The other three entries in Table 1 are derived in an analogous way, and so we omit their proofs.

We are now in a position to prove our main uniqueness theorem.

Theorem 6.5. Suppose that (2.1)-(2.4) hold, and that the coefficient $k(x, t)$ satisfies the additional regularity assumptions described in Section 5. Let $v$ and $u$ be two entropy solutions to the Cauchy problem (1.1) with initial data $v_{0}, u_{0} \in L^{\infty}(\mathbb{R})$, respectively. If $f$ satisfies the crossing condition (Assumption 6.1), and we assume the existence of traces (Assumption 6.2) then, for a.e. $t>0$

$$
\int_{-r}^{r}|v(x, t)-u(x, t)| d x \leq C \int_{-r-\left\|f_{u}\right\| t}^{r+\left\|f_{u}\right\| t}\left|v_{0}(x)-u_{0}(x)\right| d x, \quad \forall r \in \mathbb{R},
$$

for some finite constant $C>0$. If $k(x, t)$ is piecewise constant, i.e., (6.2) holds, then $C=1$.

Proof. Let $F$ be the Kružkov entropy flux defined in Lemma 5.2. Following [14], we can use the Kružkov method to prove that for any $0 \leq \psi \in \mathcal{D}\left(\mathbb{R} \times \mathbb{R}_{+}\right)$

$$
\begin{aligned}
& -\iint_{\Pi_{T}}\left(|v-u| \psi_{t}+F(k(x, t), v, u) \psi_{x}\right) d t d x \\
& \leq C \iint_{\Pi_{T}}|v-u| \psi d t d x+\sum_{m=1}^{M} \int_{0}^{\infty}\left[F(k(x, t), v, u)-s_{m}(t)|v-u|\right]_{x=\xi_{m}^{-}}^{x=\xi_{m}^{+}} \psi d t
\end{aligned}
$$




\begin{tabular}{l|ll} 
& $\hat{f}\left(k^{-}, c\right) \leq \hat{f}\left(k^{+}, c\right)$ & $\hat{f}\left(k^{-}, c\right) \geq \hat{f}\left(k^{+}, c\right)$ \\
\hline$u^{-} \leq c \leq u^{+}$ & $\hat{f}\left(k^{+}, u^{+}\right) \leq \hat{f}\left(k^{+}, c\right)$ & $\hat{f}\left(k^{-}, u^{-}\right) \leq \hat{f}\left(k^{-}, c\right)$ \\
$u^{+} \leq c \leq u^{-}$ & $\hat{f}\left(k^{-}, u^{-}\right) \geq \hat{f}\left(k^{-}, c\right)$ & $\hat{f}\left(k^{+}, u^{+}\right) \geq \hat{f}\left(k^{+}, c\right)$
\end{tabular}

TABLE 2. Entropy jump conditions in terms of $\hat{f}$.

for some constant $C$ depending on $f$, and $k_{x}$ away from discontinuities. Here the notation indicates limits from the right and left at $x=\xi_{m}$, which exist by assumption. Following the proof of Theorem 4.1 of [14], we must show that

$$
\sum_{m=1}^{M} \int_{0}^{\infty}\left[F(k(x, t), v, u)-s_{m}(t)|v-u|\right]_{x=\xi_{m}^{-}}^{x=\xi_{m}^{+}} \psi d t \leq 0 .
$$

It suffices to show that for each fixed $m \in\{1, \ldots, M\}$, and for a.e. $t \in \mathbb{R}_{+}$

$$
\begin{aligned}
S_{m}(t):=F( & \left.\left.\left.\left.k\left(\xi_{m}^{+}(t)\right), t\right), v\left(\xi_{m}^{+}(t)\right), t\right), u\left(\xi_{m}^{+}(t)\right), t\right)\right) \\
& \left.\left.\left.-F\left(k\left(\xi_{m}^{-}(t)\right), t\right), v\left(\xi_{m}^{-}(t)\right), t\right), u\left(\xi_{m}^{-}(t)\right), t\right)\right) \\
& -s_{m}(t)\left|v\left(\xi_{m}^{+}(t)\right)-u\left(\xi_{m}^{+}(t)\right)\right|+s_{m}(t)\left|v\left(\xi_{m}^{-}(t)\right)-u\left(\xi_{m}^{-}(t)\right)\right| \leq 0 .
\end{aligned}
$$

To this end, fix $m \in\{1, \ldots, M\}$, and fix a time $t \in \mathbb{R}_{+}$where all of the relevant right and left limits exist. With the simplifying notation

$$
k^{\mp}=k\left(\xi_{m}^{\mp}(t), t\right), \quad v^{\mp}=v\left(\xi_{m}^{\mp}(t), t\right), \quad u^{\mp}=u\left(\xi_{m}^{\mp}(t), t\right), \quad s_{m}(t)=s, \quad S_{m}(t)=S,
$$

$S_{m}(t)$ becomes

$$
S_{m}(t):=S=F\left(k^{+}, v^{+}, u^{+}\right)-F\left(k^{-}, v^{-}, u^{-}\right)-s\left|v^{+}-u^{+}\right|+s\left|v^{-}-u^{-}\right| .
$$

To show that $S \leq 0$, we proceed as in the proof of Theorem 4.1 of [14]. The proof in that paper applies to the case where all of the curves $\omega_{m}$ are vertical lines in the $x-t$ plane, i.e. the discontinuity speed satisifies $s \equiv 0$. We will show that the present problem can be reduced to the case where $s \equiv 0$. Keeping in mind that we are focused on a fixed point $(x, t)$ located on a fixed discontinuity curve $\omega_{m}$, we start by introducing the modified flux,

$$
\hat{f}(k, u):=f(k, u)-s u,
$$

and the associated entropy flux,

$$
\hat{F}(k, u, c):=\operatorname{sign}(u-c)(\hat{f}(k, u)-\hat{f}(k, c))=F(k, u, c)-s|u-c| .
$$

A glance at Definition 6.1, and in particular (6.1), reveals that the original flux $f$ and the modified flux $\hat{f}$ have exactly the same crossings (or lack thereof). In addition, our assumption that the crossing condition is satisfied for $f$ implies the same for $\hat{f}$. In terms of $\hat{F}$, the quantity $S$ becomes

$$
S=\hat{F}\left(k^{+}, v^{+}, u^{+}\right)-\hat{F}\left(k^{-}, v^{-}, u^{-}\right),
$$

i.e., the same quantity appearing in the proof of Theorem 4.1 of [14], except without the diffusion terms. In terms of $\hat{f}$, Table 1 of this paper becomes Table 2, which is the same as Table 1 of [14] without the diffusion terms.

Ignoring the diffusion terms that appear in [14], we can now retrace the steps of the proof of Theorem 4.1 of that paper, allowing us to complete the present proof.

By combining the convergence theorem (Theorem 4.5) and the uniqueness theorem (Theorem 6.5), we have the following well-posedness result. 
Theorem 6.6. Assuming

1. that (2.1)-(2.6) hold,

2. that the additional regularity conditions for $k$ stated in Section 5 are satisfied,

3. that $f$ satisfies the crossing condition (Assumption 6.1), and

4. that traces exist (Assumption 6.2),

then a unique entropy solution to the Cauchy problem (1.1) exists, and the solution depends continuously (in the $L^{1}$ norm) on the initial data. The entire computed sequence of approximations $\left\{u^{\Delta}\right\}$ generated by the Lax-Friedrichs scheme given by (2.7), (2.8), (2.11), (5.2) converges to the unique entropy solution $u$.

Remark 6.7. If $k(x, t)$ is piecewise constant, i.e., (6.2) holds, then assumption 4 . in Theorem 6.6 is automatically fulfilled thanks to Lemma 6.2 .

\section{A FINAL REMARK ABOUT UNIQUENESS}

Our uniqueness theorem (Theorem 6.5) was established under the so-called crossing condition (Assumption 6.1). If there is a flux crossing (or in fact, just an intersection of the two flux curves) associated with a jump in $k$, say at $\xi_{m}$, then it is possible for the solution $u$ to be continuous across the jump, i.e., $u_{m}^{-}=u_{m}^{+}$. In that case, both the Rankine-Hugoniot condition (6.11) and the entropy jump condition (6.12) hold trivially, as do the conditions listed in Table 1 . We emphasize that our entropy theory does not impose any additional condition on the solution in this situation, and that such solutions are thus admissible under our definition of entropy solution. In this regard, our entropy theory is somewhat different from that of Adimurthi, Gowda, and Jaffre [1], who require that a certain characteristic condition hold even if the solution $u$ is continuous at a jump in $k$. In particular, there are situations where their entropy solution theory rules out continuous solutions that are admissible under our entropy theory. The main application discussed in [1] is flow in porous media, and evidently a more compressive solution is appropriate in that setting, based on physical considerations. One application that has been of great interest to us is the clarifier-thickener model $[2,3]$. In this setting, where we have a jump in $k$ due to a source term giving rise to diverging flows, our entropy solution theory seems appropriate.

We defer to [15] a more detailed discussion of the relationship between our entropy condition and the characteristic condition mentioned above. For now, we merely note that that the characteristic condition of Adimurthi, Gowda, and Jaffre is enforced by our entropy conditions, except in the case above where the flux curves intersect and $u_{m}^{-}=u_{m}^{+}$. In particular, if the flux curves $f\left(k_{m}^{-}, u\right)$ and $f\left(k_{m}^{+}, u\right)$ do not intersect, our entropy conditions imply the characteristic condition of Adimurthi, Gowda, and Jaffre. Finally, let us mention that Kaasschieter [10] proposes essentially the same characteristic condition as Adimurthi, Gowda, and Jaffre, but he assumes that the flux curves $f\left(k_{m}^{-}, u\right)$ and $f\left(k_{m}^{+}, u\right)$ do not intersect, and so his entropy theory is consistent with the one proposed herein.

\section{REFERENCES}

[1] Adimurthi, J. Jaffre, and G. Gowda. Godunov-type methods for conservation laws with a flux function discontinuous in space. SIAM J. Numer. Anal. To appear.

[2] R. Bürger, K. H. Karlsen, and S. Berres. Central schemes and systems of conservation laws with discontinuous coefficients modeling gravity separation of polydisperse suspensions. J. Comp. Appl. Math., 164-165: 53-80, 2004

[3] R. Bürger, K. H. Karlsen, N. H. Risebro, and J. D. Towers. Well-posedness in $B V_{t}$ and convergence of a difference scheme for continuous sedimentation in ideal clarifier-thickener units. Numer. Math., 97(1):25-65, 2004.

[4] G.-Q. Chen. Compactness methods and nonlinear hyperbolic conservation laws. In Some current topics on nonlinear conservation laws, pages 33-75. Amer. Math. Soc., Providence, RI, 2000.

[5] G. M. Coclite and N. H. Risebro. Conservation laws with time dependent discontinuous coefficients. Preprint available at the URL http://www.math.ntnu.no/conservation/, 2002.

[6] M. G. Crandall and A. Majda. Monotone difference approximations for scalar conservation laws. Math. Comp., 34(149):1-21, 1980.

[7] R. J. DiPerna. Convergence of approximate solutions to conservation laws. Arch. Rat. Mech. Anal., 82:27-70, 1983. 
[8] T. Gimse and N. H. Risebro. Solution of the Cauchy problem for a conservation law with a discontinuous flux function. SIAM J. Math. Anal., 23(3):635-648, 1992.

[9] J. M.-K. Hong. Part I: An extension of the Riemann problems and Glimm's method to general systems of conservation laws with source terms. Part II: A total variation bound on the conserved quantities for a generic resonant nonlinear balance laws. PhD thesis, University of California, Davis, 2000.

[10] E. F. Kaasschieter. Solving the Buckley-Leverett equation with gravity in a heterogeneous porous medium. Comput. Geosci., 3(1):23-48, 1999.

[11] K. H. Karlsen, C. Klingenberg, and N. H. Risebro. A relaxation scheme for conservation laws with discontinuous coefficients. Math. Comp., 73(247): 1235-1259, 2004.

[12] K. H. Karlsen, N. H. Risebro, and J. D. Towers. On a nonlinear degenerate parabolic transport-diffusion equation with a discontinuous coefficient. Electron. J. Differential Equations, No. 93, 23 pp. (electronic), 2002.

[13] K. H. Karlsen, N. H. Risebro, and J. D. Towers. Upwind difference approximations for degenerate parabolic convection-diffusion equations with a discontinuous coefficient. IMA J. Numer. Anal., 22(4):623-664, 2002.

[14] K.H. Karlsen, N.H. Risebro And J.D. Towers, $L^{1}$ stability for entropy solutions of nonlinear degenerate parabolic convection-diffusion equations with discontinuous coefficients, Skr. K. Nor. Vid. Selsk, No. 3, 49 pp., 2003

[15] K. H. Karlsen, N. H. Risebro, and J. D. Towers. Entropy conditions and uniqueness for conservation laws with crossing flux discontinuities. Manuscript in preparation.

[16] R. A. Klausen and N. H. Risebro. Stability of conservation laws with discontinuous coefficients. J. Differential Equations, 157(1):41-60, 1999.

[17] C. Klingenberg and N. H. Risebro. Convex conservation laws with discontinuous coefficients. Existence, uniqueness and asymptotic behavior. Comm. Partial Differential Equations, 20(11-12):1959-1990, 1995.

[18] C. Klingenberg and N. H. Risebro. Stability of a resonant system of conservation laws modeling polymer flow with gravitation. J. Differential Equations, 170(2):344-380, 2001.

[19] P. D. Lax. Shock waves and entropy. In E. Zarantonello, editor, Contributions to Nonlinear Functional Analysis, pages 603-634. Academic Press, New York, 1971.

[20] L. W. Lin, B. J. Temple, and J. H. Wang. A comparison of convergence rates for Godunov's method and Glimm's method in resonant nonlinear systems of conservation laws. SIAM J. Numer. Anal., 32(3):824-840, 1995.

[21] L. W. Lin, B. J. Temple, and J. H. Wang. Suppression of oscillations in Godunov's method for a resonant non-strictly hyperbolic system. SIAM J. Numer. Anal., 32(3):841-864, 1995.

[22] Y. Lu. Hyperbolic conservation laws and the compensated compactness method, volume 128 of Chapman $\mathcal{B}$ Hall/CRC Monographs and Surveys in Pure and Applied Mathematics. Chapman \& Hall/CRC, Boca Raton, FL, 2003.

[23] F. Murat. Compacité par compensation. Ann. Scuola Norm. Sup. Pisa Cl. Sci. (4), 5(3):489-507, 1978.

[24] F. Murat. Compacité par compensation. II. In Proceedings of the International Meeting on Recent Methods in Nonlinear Analysis (Rome, 1978), pages 245-256, Bologna, 1979. Pitagora.

[25] F. Murat. L'injection du cône positif de $H^{-1}$ dans $W^{-1, q}$ est compacte pour tout $q<2$. J. Math. Pures Appl. (9), 60(3):309-322, 1981.

[26] H. Nessyahu and E. Tadmor. Nonoscillatory central differencing for hyperbolic conservation laws. J. Comput. Phys., 87(2):408-463, 1990.

[27] D. N. Ostrov. Solutions of Hamilton-Jacobi equations and scalar conservation laws with discontinuous spacetime dependence. J. Differential Equations, 182(1):51-77, 2002.

[28] N. Seguin and J. Vovelle. Analysis and approximation of a scalar conservation law with a flux function with discontinuous coefficients. Preprint 2002.

[29] E. Tadmor. Approximate solutions of nonlinear conservation laws. In A. Quarteroni, editor, Lecture notes of the 1997 C.I.M.E. course in Cetraro (Cosenza), Italy. Springer Verlag, 1997.

[30] L. Tartar. Compensated compactness and applications to partial differential equations. In Nonlinear analysis and mechanics: Heriot-Watt Symposium, Vol. IV, pages 136-212. Pitman, Boston, Mass., 1979.

[31] L. Tartar. The compensated compactness method applied to systems of conservation laws. In Systems of nonlinear partial differential equations (Oxford, 1982), volume 111 of NATO Adv. Sci. Inst. Ser. C Math. Phys. Sci., pages 263-285. Reidel, Dordrecht, 1983.

[32] B. Temple. Global solution of the Cauchy problem for a class of $2 \times 2$ nonstrictly hyperbolic conservation laws. Adv. in Appl. Math., 3(3):335-375, 1982.

[33] J. D. Towers. Convergence of a difference scheme for conservation laws with a discontinuous flux. SIAM J. Numer. Anal., 38(2):681-698, 2000.

[34] J. D. Towers. A difference scheme for conservation laws with a discontinuous flux - the nonconvex case. SIAM J. Numer. Anal., 39(4):1197-1218, 2001.

[35] A. Tveito and R. Winther. The solution of nonstrictly hyperbolic conservation laws may be hard to compute. SIAM J. Sci. Comput., 16(2):320-329, 1995.

[36] A. Vasseur. Strong traces for solutions of multidimensional scalar conservation laws. Arch. Ration. Mech. Anal., 160(3):181-193, 2001. 
(Kenneth Hvistendahl Karlsen)

Department of Mathematics

UNIVERSITY OF BERGEN

Johs. BRUNSGT. 12

N-5008 Bergen, Norway

AND

Centre of Mathematics for Applications

Department of Mathematics

UNIVERSITY OF OSLO

P.O. BOX 1053, BLINDERN

N-0316 Oslo, Norway

E-mail address: kennethk@mi.uib.no

URL: http://www.mi.uib.no/ kennethk/

(John D. Towers)

MiraCosta College

3333 Manchester Avenue

CARDiff-BY-The-SeA, CA 92007-1516, USA

E-mail address: jtowers@cts.com

$U R L$ : www.miracosta.cc.ca.us/home/jtowers/ 